\title{
SLIT2/ROBO signaling in tumor-associated microglia and macrophages drives glioblastoma immunosuppression and vascular dysmorphia
}

\author{
Luiz H. Geraldo, ${ }^{1,2}$ Yunling Xu, ${ }^{1}$ Laurent Jacob, ${ }^{1}$ Laurence Pibouin-Fragner, ${ }^{1}$ Rohit Rao, ${ }^{3}$ Nawal Maissa, ${ }^{1}$ Maïté Verreault, ${ }^{4}$ \\ Nolwenn Lemaire, ${ }^{4}$ Camille Knosp, ${ }^{1}$ Corinne Lesaffre, ${ }^{1}$ Thomas Daubon, ${ }^{5,6}$ Joost Dejaegher, ${ }^{7,8}$ Lien Solie, ${ }^{7,8}$ Justine Rudewicz, ${ }^{5,6}$ \\ Thomas Viel, ${ }^{1}$ Bertrand Tavitian, ${ }^{1}$ Steven De Vleeschouwer,,${ }^{7,8}$ Marc Sanson, ${ }^{4,9}$ Andreas Bikfalvi, ${ }^{5,6}$ Ahmed Idbaih, ${ }^{4}$ Q. Richard Lu, ${ }^{3}$ \\ Flavia R.S. Lima, ${ }^{2}$ Jean-Leon Thomas, ${ }^{4,10}$ Anne Eichmann, ${ }^{1,11,12}$ and Thomas Mathivet ${ }^{1}$ \\ 'Université de Paris, Paris Cardiovascular Research Center, INSERM, Paris, France. ${ }^{2}$ Biomedical Sciences Institute, Federal University of Rio de Janeiro, Brazil. ${ }^{3}$ Brain Tumor Center, Cincinnati Children's Hospital \\ Medical Center, Cincinnati, Ohio, USA. ${ }^{4}$ Sorbonne Université, INSERM U1127, CNRS UMR 7225, Institut du Cerveau, ICM, AP-HP, Hôpitaux Universitaires La Pitié Salpêtrière-Charles Foix, Service de Neurologie \\ 2-Mazarin, Paris, France. ${ }^{5}$ NSERM U1029, ${ }^{6}$ Université de Bordeaux, Pessac, France. ${ }^{7}$ Department of Neurosciences and ${ }^{8}$ Department of Neurosurgery, UZ Leuven, Leuven, Belgium. ${ }^{9}$ Onconeurotek Tumor \\ Bank, Institut du Cerveau et de la Moelle épinière-ICM, Paris, France. ${ }^{10}$ Department of Neurology, ${ }^{11}$ Cardiovascular Research Center, Department of Internal Medicine, and ${ }^{12}$ Department of Cellular and \\ Molecular Physiology, Yale University School of Medicine, New Haven, Connecticut, USA
}

SLIT2 is a secreted polypeptide that guides migration of cells expressing Roundabout 1 and 2 (ROBO1 and ROBO2) receptors. Herein, we investigated SLIT2/ROBO signaling effects in gliomas. In patients with glioblastoma (CBM), SLIT2 expression increased with malignant progression and correlated with poor survival and immunosuppression. Knockdown of SLIT2 in mouse glioma cells and patient-derived CBM xenografts reduced tumor growth and rendered tumors sensitive to immunotherapy. Tumor cell SLIT2 knockdown inhibited macrophage invasion and promoted a cytotoxic gene expression profile, which improved tumor vessel function and enhanced efficacy of chemotherapy and immunotherapy. Mechanistically, SLIT2 promoted microglia/macrophage chemotaxis and tumor-supportive polarization via ROBO1- and ROBO2-mediated PI3K- $\gamma$ activation. Macrophage Robo1 and Robo2 deletion and systemic SLIT2 trap delivery mimicked SLIT2 knockdown effects on tumor growth and the tumor microenvironment (TME), revealing SLIT2 signaling through macrophage ROBOs as a potentially novel regulator of the CBM microenvironment and immunotherapeutic target for brain tumors.

\section{Introduction}

Malignant gliomas are the most common primary brain tumors $(1,2)$. Among those, glioblastoma (GBM; WHO grade IV glioma) is the most frequent and aggressive tumor, accounting for more than $50 \%$ of gliomas, and with poor patient prognosis (3). GBMs are molecularly heterogeneous and invasive, angiogenic, and proliferative tumors that are largely resistant to current therapies (4).

Tumor-associated microglia and macrophages (TAMs) are the most abundant cells in the GBM microenvironment, composing up to $25 \%$ of the tumor mass (5-7). TAMs are key drivers of GBM immunosuppression and pathological angiogenesis (7). TAMs inhibit $\mathrm{T}$ cell responses in the GBM microenvironment by favoring regulatory $\mathrm{T}$ cells and suppressing antitumor $\mathrm{T}$ cell responses (8-11), thereby limiting the efficacy of currently available T celloriented immunotherapies in GBM $(4,12-14)$. TAM-derived signaling also contributes to vascular dysmorphia, and drives blood vessel dilation and leakiness in the GBM microenvironment (15,

Authorship note: $\mathrm{AE}$ and TM contributed equally to this work. Conflict of interest: The authors have declared that no conflict of interest exists. Copyright: () 2021, American Society for Clinical Investigation. Submitted: June 5, 2020; Accepted: June 22, 2021; Published: August 16, 2021 Reference information: J Clin Invest. 2021;131(16):e141083. https://doi.org/10.1172/JCl141083.
16). Nonuniform oxygen delivery via dysmorphic and leaky tumor vessels leads to hypoxia, which upregulates angiogenic factors that induce more dysfunctional vessels, thereby preventing the delivery of cytotoxic agents to kill tumor cells $(4,17)$. The mechanisms by which TAMs promote vessel dysmorphia and immune evasion are as yet incompletely understood, and the means to prevent them are not available $(7,18,19)$.

SLITs are evolutionary conserved secreted polypeptides that bind to transmembrane Roundabout (ROBO) receptors $(20,21)$. In mammals, 3 SLIT ligands (SLIT1-3) signal via 2 ROBO receptors, ROBO1 and ROBO2 (22). SLIT ligands bind via the second leucine-rich repeat region (D2) to the Ig1 domain of ROBO1 and ROBO2 (23), while mammalian ROBO3 and ROBO4 lack the SLIT binding residues and do not bind SLITs $(24,25)$. SLIT binding triggers recruitment of adaptor proteins to the ROBO cytoplasmic domain that modulate the cytoskeleton, in turn regulating cell migration, adhesion, and proliferation $(22,26,27)$.

SLIT/ROBO signaling, which regulates pathfinding of commissural axons and motor coordination between the left and right sides of the body, was discovered in the developing nervous system to be a guidance cue for axonal growth cones (20, 21). It is now known that SLIT/ROBO signaling controls several additional biological processes, including angiogenesis and immune cell migration. 

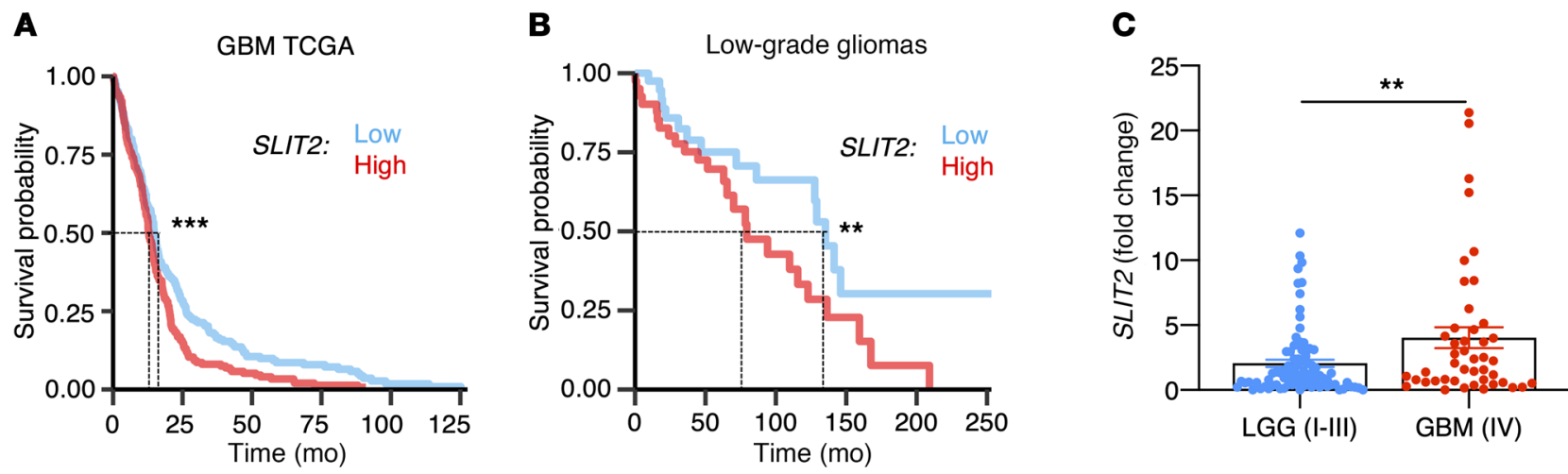

D

\section{E}
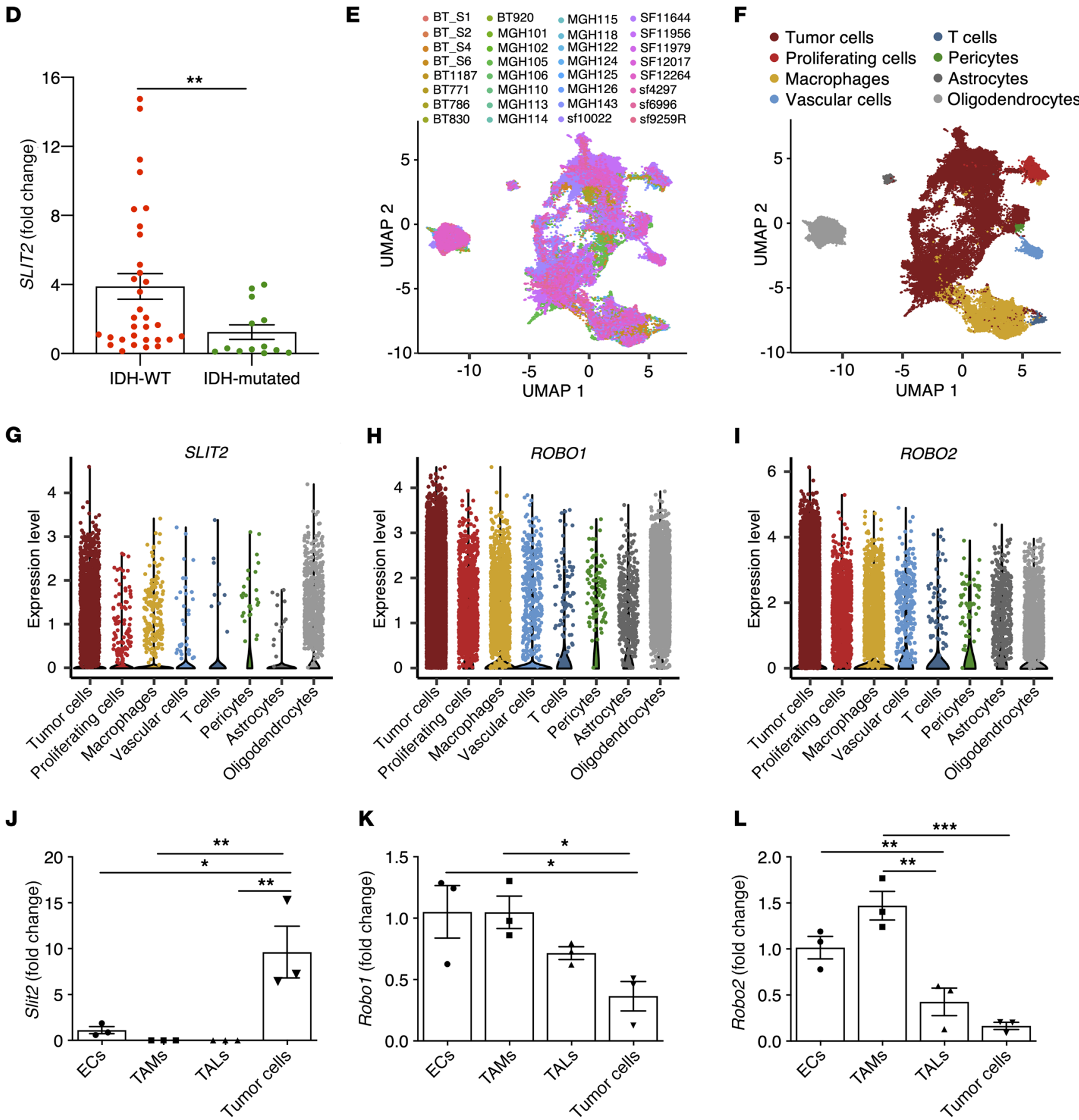
Figure 1. Slit2 expression correlates with glioma aggressiveness and poor patient prognosis. (A) In silico analysis of TCGA glioblastoma Agilent-4502A patient data set $(n=244$ high, 244 low for patients expressing Slit2; OS 12.9 months for high expression and 15.1 months for low expression, log-rank test). (B) Survival analysis of patients with LGG (grades I to III) grouped by their levels of SLIT2 expression ( $n=41$ high and 41 low; OS 79.4 months for high expression and 135.2 months for low expression, log-rank test). (C) SLIT2 qPCR expression in samples from patients with glioma shown in B (CBM, $n=45$; LGG, $n=84$; Student's $t$ test). (D) SLIT2 qPCR expression in grades III and IV glioma patient samples classified by their IDH-1/2 status (IDH-WT, $n=51$; IDH-mutated, $n=34$; Mann-Whitney $U$ test). ( $E$ and $\mathbf{F}$ ) UMAP plots of scRNAseq of 32 patients with GBM showing different samples (E) and clustering of the different cell types in the GBM microenvironment (F). (G-I) Expression plots of SLIT2 (G), ROBO1 (H), and ROBO2 (I) in scRNAseq data from E. (J-L) qPCR analysis of Slitz (J), Robo1 (K), and Robo2 (L) expression in ECs, TAMs, TALs, and tumor cells FACS-sorted from late-stage CT-2A mice glioblastomas ( $n=3$ independent tumors, day 21 after implantation, 1-way ANOVA). All data are mean \pm SEM. ${ }^{*} P<0.05,{ }^{* *} P<0.01,{ }^{* *} P<0.001$.

In endothelial cells, SLIT2 activation of ROBO1 and ROBO2 signaling promotes retinal and bone angiogenesis by driving tip cell migration and polarization (28-31). In the immune system, SLITs have been described as chemo-attractive for neutrophils (32) and chemorepellent for lymphocytes and dendritic cells (3336). In macrophages, SLIT/ROBO signaling prevented macropinocytosis and cytotoxic polarization (37).

In tumor contexts, SLIT2 exerts a proangiogenic role (38-40), and has been reported to enhance tumor cell aggressiveness and migration (41-45), metastatic spread $(40,46)$, and therapy resistance (47), particularly in colorectal cancer, pancreatic cancer, and osteosarcoma. Nevertheless, other studies reported a tumor suppressive role for SLIT2/ROBO signaling in lung and breast cancers (48-50). In the context of GBM, some studies suggested that SLIT2 signaling could inhibit tumor growth (51-53), while in others SLIT/ ROBO signaling correlated with more aggressive GBM behavior $(54,55)$. Given the various and context-dependent effects of SLIT/ ROBO signaling in cancer, it remained unclear if this pathway could be used therapeutically to prevent cancer growth.

We showed here that high SLIT2 expression in patients with GBM and in mouse models induced TAM accumulation and vascular dysmorphia, and that SLIT2 knockdown in glioma cells and systemic SLIT2 inhibition with a ligand trap normalized the tumor microenvironment (TME) by preventing TAM tumor-supportive polarization and angiogenic gene expression. As a result, antitumor immune responses and tumor perfusion were enhanced, and efficacy of temozolomide-based (TMZbased) chemotherapy and $\mathrm{T}$ cell-based immunotherapy were increased. Inducible genetic deletion of Robo1 and Robo2 in macrophages was sufficient to normalize the TME and enhanced response to immunotherapy, revealing a potentially novel macrophage-based immunotherapy approach for GBM.

\section{Results}

SLIT2 expression correlated with poor prognosis in patients with glioma. The Cancer Genome Atlas (TCGA) Agilent-4502A microarray data analysis using median expression as cutoff showed that high SLIT 2 expression was significantly associated with decreased survival in patients with GBM (Figure 1A, overall survival [OS],
12.9 months for high expression and 15.1 months for low expression). Analysis of other TCGA data sets confirmed an association between high SLIT2 expression and decreased survival in patients with GBM, even though this association did not reach statistical significance within all the data sets (Supplemental Figure 1, A and B; supplemental material available online with this article; https://doi.org/10.1172/JCI141083DS1). Expression of the other SLIT family members and $R O B O$ receptors was not associated with worse prognosis (Supplemental Figure 1, C-F).

Analysis of a cohort of patients with primary glioma (129 patients: 84 low grade gliomas [LGGs] and $45 \mathrm{GBMs}$ ) also demonstrated correlation between high SLIT2 expression and worse prognosis in both LGGs and GBMs (Figure 1B, OS for LGG: 79.2 months for high expression and 135.2 months for low expression; Supplemental Figure 1G, OS for GBM: 15 months for high expression and 16.5 months for low expression). Analysis from TCGA LGG data sets showed a trend toward an association between higher SLIT2 expression and reduced survival, but these results were not statistically significant (Supplemental Figure 1H, OS 75 months for high expression and 94.5 months for low expression, log-rank test).

Further analysis of RNA sequencing data demonstrated higher SLIT2 expression in the most aggressive and angiogenic mesenchymal GBM subtype (56) and lower expression in classical GBMs (Supplemental Figure 1I). High SLIT2 expression was also associated with poor survival in patients with mesenchymal GBM in this cohort (Supplemental Figure 1J, OS 10.4 months for high expression and 17.9 months for low expression, log-rank test). Finally, quantitative PCR (qPCR) analysis of patient samples also revealed higher expression levels of SLIT2 in WHO grade IV GBM compared with patients with WHO grade I, II, and III glioma (Figure 1C), while expression of other SLITs and ROBOs was not changed between glioma grades (Supplemental Figure 1, K-N). Expression levels of SLIT1 and SLIT3 were significantly lower compared with SLIT2 in patients with GBM (Supplemental Figure 1O).

Isocitrate dehydrogenase 1 and 2 (IDH-1/2) mutations are known prognostic factors in malignant gliomas. Patients with grade III gliomas and no IDH mutations (IDH-WT) have comparable prognoses to those of patients with GBM, while patients with IDH mutations have better survival prognosis (57-59). We compared patients with glioma classified by IDH-status, and observed increased SLIT2 expression in patients with IDH-WT tumors in either grade III and IV gliomas (Figure 1D) or in all gliomas (Supplemental Figure 1P).

To determine the source of SLIT2 in the GBM microenvironment, we analyzed single-cell RNA sequencing (scRNAseq) data from patients with GBM (Figure 1, E and F). The majority of the cells expressing SLIT2 mRNA were cancer cells and oligodendrocytes (Figure 1G), while ROBO1 and ROBO2 were mostly expressed in tumor cells but also detected in other cell types in the TME, particularly in TAMs (Figure $1, \mathrm{H}$ and I).

We next generated a mouse model of GBM by intracerebral inoculation of syngeneic CT-2A mouse glioma tumor cells expressing green fluorescent protein (GFP) into adult C57BL/6 mice $(16,60)$. Expression of Slit ligands and their Robo receptors was tested 21 days after tumor cell inoculation by qPCR on FACS-sorted tumor cells $\left(\mathrm{GFP}^{+}\right)$, endothelial cells (ECs, CD31+), 
A

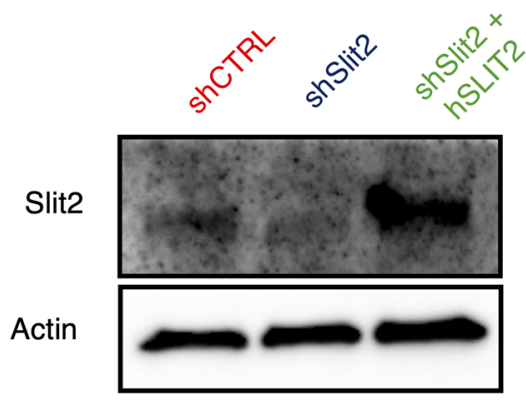

B

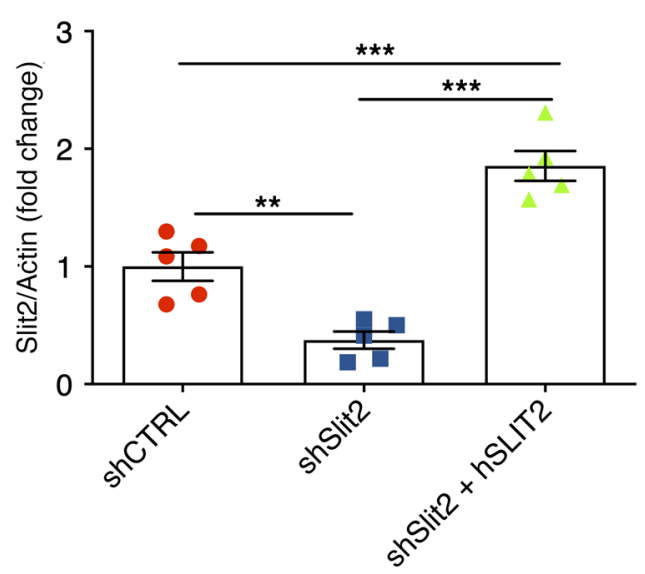

C

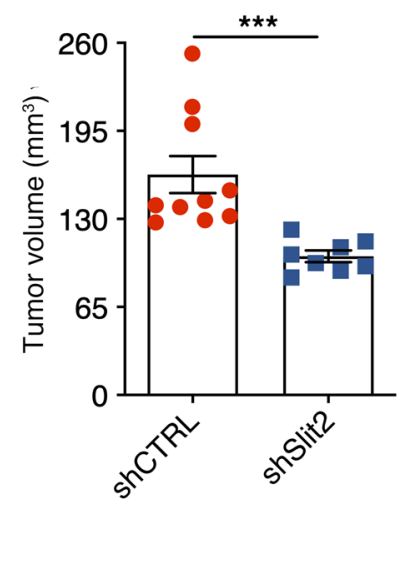

D
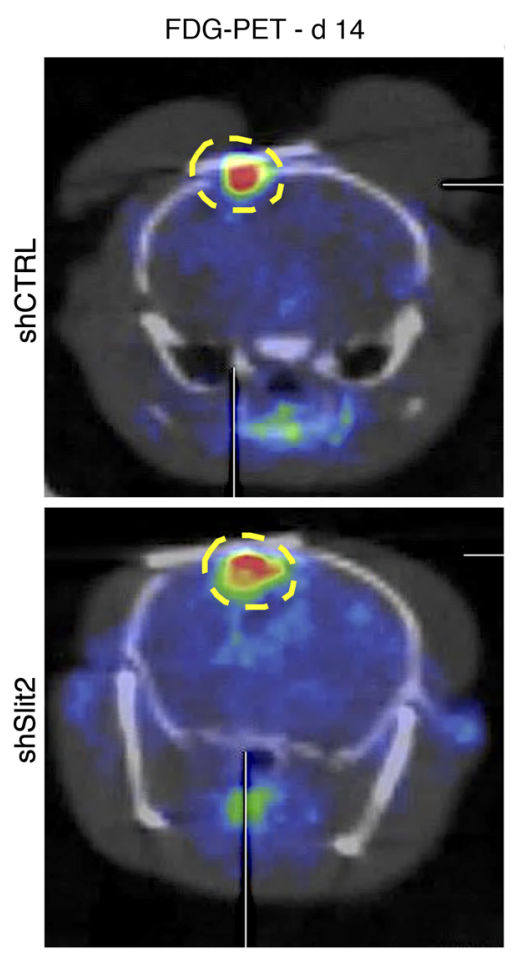

G

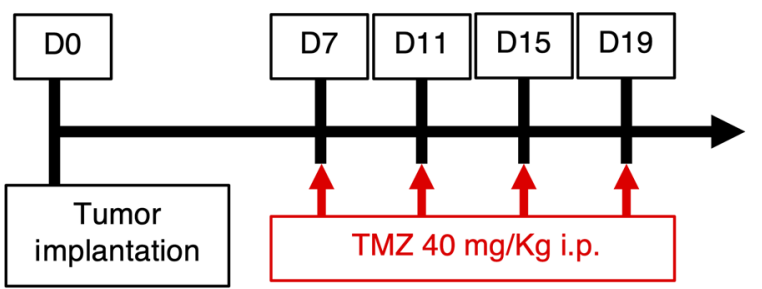

FDG-PET - d 21
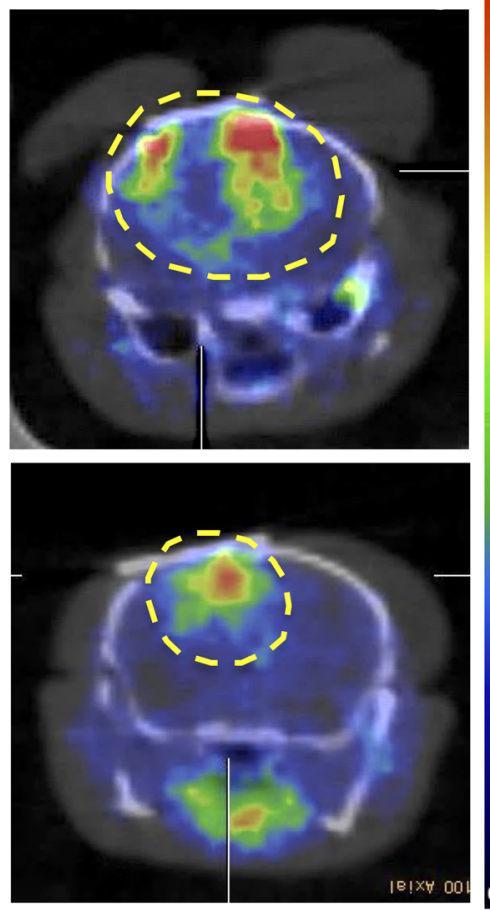

H
E

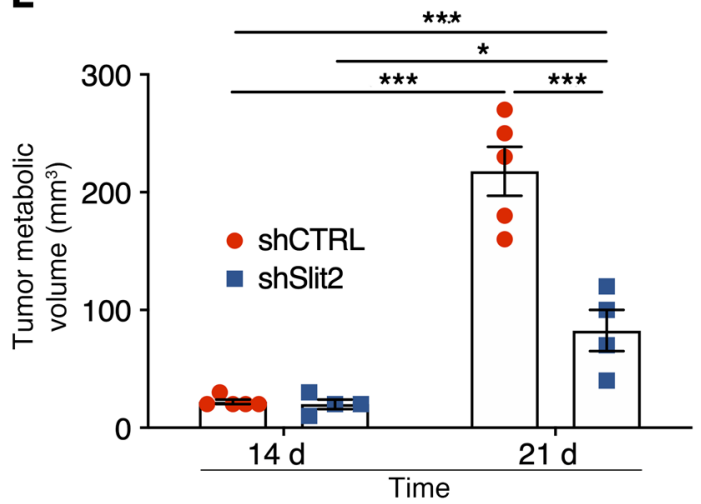

$\mathbf{F}$

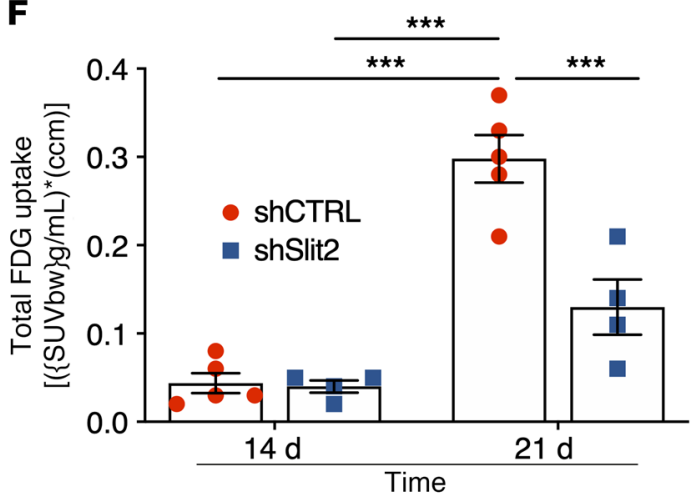

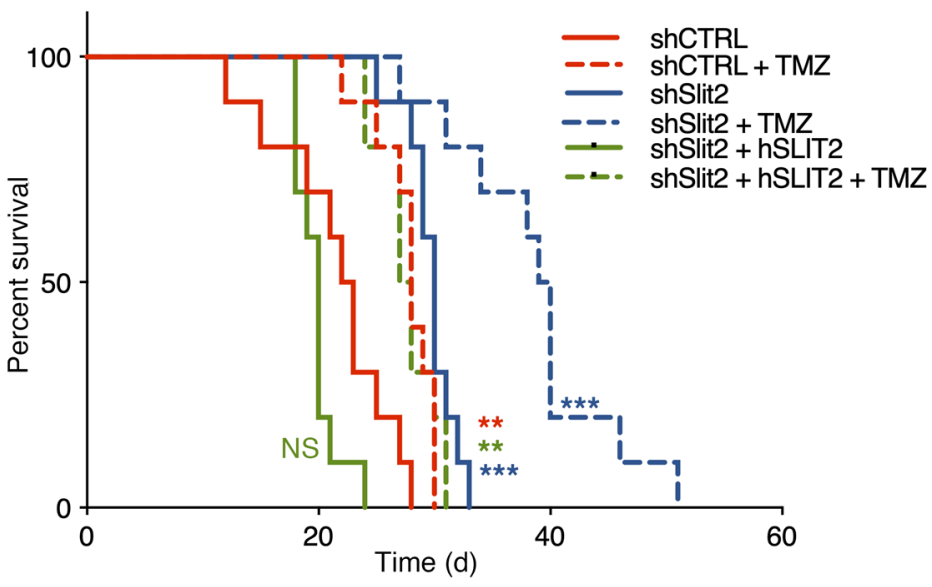


Figure 2. Slit2 promotes glioblastoma growth and resistance to TMZ. (A and B) Western blot analysis (A) and quantification (B) of Slit2 expression in shCTRL, shSlit2, and shSlit2 + hSLIT2 CT-2A cells ( $n=5$, 1-way ANOVA). (C) Tumor volume quantification at 21 days ( $n=10$ for shCTRL and $n=8$ for shSlit2, Student's $t$ test). (D) FDG-PET imaging over CT-2A shCTRL and shSlit2 glioma growth ( $n=5$ shCTRL and $n=4$ shSlit2). (E and F) Quantification of tumor metabolic volume (E) and total tumor glucose uptake (F) from D ( $n=5$ for shCTRL and $n=4$ for shSlit2, 1-way ANOVA). (C) Survival trial design: 8-week-old mice were engrafted with CT-2A shCTRL, shSlit2, or shSlit2 + hSLIT2 spheroids and randomly assigned to vehicle or TMZ treatment ( $40 \mathrm{mg} / \mathrm{kg}$ on days $7,11,15$, and 19 after tumor implantation). (H) Survival curves of the mice in $\mathbf{G}(n=10$ mice per group, OS 22.5 days for shCTRL, 28 days for shCTRL + TMZ, 30 days for shSlit2, 39.5 days for shSlit2 + TMZ, 20 days for shSlit2 + hSLIT2, and 27 days for shSlit2 + hSLIT2 + TMZ; multiple comparisons log-rank test). Data are mean \pm SEM. ${ }^{*} P<0.05,{ }^{* *} P<0.01,{ }^{* * *} P<0.001$.

TAMs $\left(\mathrm{CD} 45^{+} \mathrm{CD} 11 \mathrm{~b}^{+} \mathrm{CD} 3^{-}\right)$, and tumor-associated $\mathrm{T}$ lymphocytes (TALs, $\mathrm{CD}^{4} 5^{+} \mathrm{CD} 11 \mathrm{~b}^{-} \mathrm{CD}^{+}$). The major source of Slit2 ligands were the tumor cells themselves (Figure 1J). By contrast, Robo1 and Robo2 receptors were mainly expressed by ECs and recruited TAMs and TALs (Figure 1, K and L). Slit1 and Slit3 expression levels in mouse tumor cells were much lower when compared with Slit2 (Supplemental Figure 1Q). These data suggested that interactions between tumor cell-derived SLIT2 and stromal cells expressing ROBOs could affect GBM growth.

Slit2 silencing slowed GBM growth and increased TMZ sensitivity. To determine if tumor cell-derived Slit2 affected GBM growth, we infected CT-2A and GL261 glioma cells with lentivirus encoding GFP-tagged control scrambled shRNA (shCTRL) or Slit2 targeting shRNA (shSlit2) alone or combined with an shRNA-resistant human SLIT2 construct (shSlit2 + hSLIT2). Slit2 knockdown significantly decreased Slit2 protein and mRNA expression, while shSlit2 + hSLIT2 cells expressed more Slit2 than controls (Figure 2, A and B and Supplemental Figure 2, A-F). Expression of other Slits or Robo1 and 2 was not altered (data not shown). In vitro growth rates of shCTRL and shSlit2 CT2A and GL261 knockdown cells were similar (Supplemental Figure 2, G and H). Slit2 did not induce tumor cell chemotaxis in a transwell chamber assay (Supplemental Figure 2, I and J). Nevertheless, migration of shSlit2 cells toward a serum gradient in the lower chamber was reduced (Supplemental Figure 2, K and L).

Individual $250-\mu \mathrm{m}$ diameter tumor cell spheroids were implanted through cranial windows into Tomato-fluorescence reporter mice $\left(\mathrm{ROSA}^{\mathrm{mT} / \mathrm{mG}}\right)$ and followed longitudinally. Compared with shCTRL, Slit2 knockdown tumors exhibited reduced volumes after 21 days (Figure 2C). F-18 fluorodeoxyglucose (FDG)PET imaging showed that tumor metabolic volume and FDG total uptake were similar between shSlit2 and shCTRL at 14 days, but reduced in shSlit2 tumors at 21 days (Figure 2, D-F), demonstrating that Slit2 knockdown delayed tumor growth in vivo.

We investigated if Slit2 knockdown affected survival in combination with low-dose chemotherapy with the DNA alkylating agent TMZ, a classical treatment for GBM (Figure 2G). Compared with shCTRL, Slit 2 knockdown increased overall survival of tumor-bearing mice, while Slit2 overexpression tended to decrease survival (Figure 2H, OS 22.5 days for shCTRL, 30 days for shSlit2, and 20 days shSlit $2+$ hSLIT2). TMZ treatment further increased
OS of shSlit2 glioma-bearing mice (Figure 2H, OS 28 days TMZ for shCTRL+ TMZ, 39 days for shSlit2 + TMZ, and 27.5 days for shSlit2 + hSLIT2 + TMZ). shSlit2 did not affect TMZ sensitivity of tumor cells in vitro (Supplemental Figure 2M), but significantly increased TMZ-induced $\mathrm{pH}_{2} \mathrm{AX}^{+}$double-strand DNA breaks in tumors in vivo (Supplemental Figure 2, N and O), suggesting that changes in the TME might contribute to enhanced TMZ sensitivity in vivo.

SLIT2 silencing slowed GBM growth and invasiveness in a patient-derived xenograft (PDX) model. To determine whether SLIT2 had similar effects on human GBM tumors, we used N150460 patient-derived GBM cells that were established from a biopsy and grown as tumor spheres. We infected these cells with lentivirus encoding a luciferase reporter and GFP-tagged shCTRL or shSLIT2. SLIT2 knockdown significantly decreased SLIT2 protein and mRNA expression without altering expression of other SLITs or ROBO1 and ROBO2 (Supplemental Figure $3, A-G)$. In vitro growth rates of shCTRL and shSLIT2 cells and sensitivity to TMZ were similar (Supplemental Figure 3, H and I). SLIT2 did not induce tumor cell chemotaxis in a transwell chamber assay, but migration of shSLIT2 cells toward a serum gradient in the lower chamber was reduced (Supplemental Figure 3, J and K). Next, we analyzed sphere formation and observed that shCTRL and shSLIT2 cells formed similar numbers of spheres after 48 hours in culture, but the size of shSLIT2 spheres was reduced when compared with shCTRL (Supplemental Figure 3, L and M). Analysis of tumor sphere invasion in fibrin gels showed that shSLIT2 decreased spheroid invasion after 24 and 48 hours in culture when compared with shCTRL (Supplemental Figure 3, N and O).

To determine the effect of shSLIT2 on human GBM growth, we implanted shCTRL and shSLIT2 N15-0460 cells in Hsd: Athymic Nude-Foxn1nu mice and followed tumor growth by bioluminescence analysis every 2 weeks after tumor implantation. At 170 days after tumor implantation, $80 \%$ of the mice injected with shCTRL cells developed tumors, while only $20 \%$ of shSLIT2injected mice had tumors (Supplemental Figure 4A). Analysis of the bioluminescence curves of shCTRL and shSLIT2 tumors demonstrated that more mice developed tumors in the shCTRL group and that the shCTRL tumors were bigger than the shSLIT2 tumors (Supplemental Figure 4, B and C). Histological analysis of $\mathrm{GFP}^{+}$tumor cells on vibratome sections 170 days after tumor implantation showed that shCTRL cells either developed tumor masses or spread throughout the entire brain, while shSLIT2 cells remained restrained to the injection site or migrated through the corpus callosum, but did not form tumor masses (Supplemental Figure 4, D and E). SLIT2 shRNA also reduced the expression of SOX2 and PML involved in GBM tumor cell malignancy (refs. 55, 61, 62 and Supplemental Figure 4, F-H).

Slit 2 knockdown improved tumor vessel function. To determine if tumor-secreted SLIT2 affected the GBM microenvironment, we used 2-photon in vivo imaging of red fluorescence $\mathrm{ROSA}^{\mathrm{mT} / \mathrm{mG}}$ mice. We observed that blood vessels in shCTRL CT2A and GL261 tumors became abnormally enlarged and lost branching points between day 14 and day 21, while shSlit2 tumor vessels dilated less and remained more ramified (Figure 3, A-C and Supplemental Figure 5, A-D). Conversely, tumor vessels from SLIT2-overexpressing mice dilated and lost branchpoints earlier, at day 18 after 
A
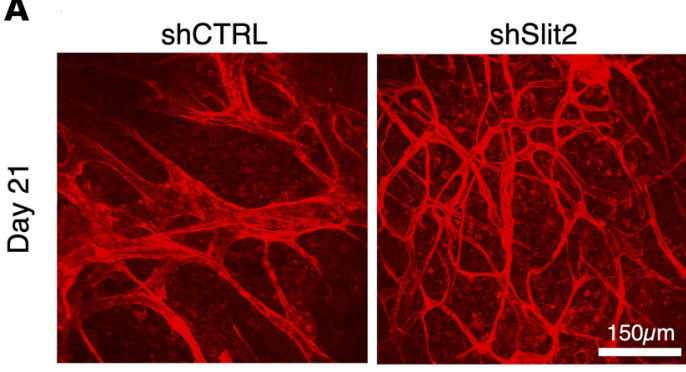

D

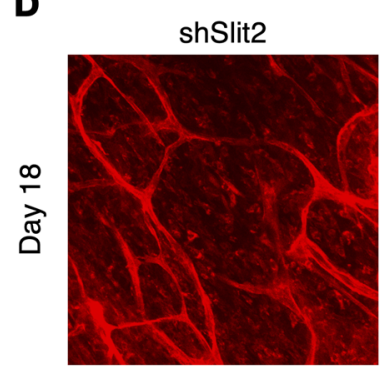

shSlit2 + hSLIT2

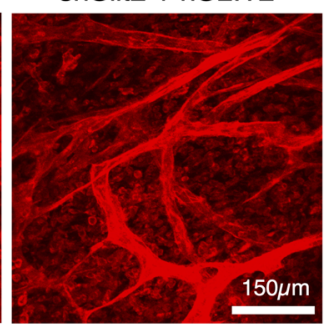

G

Tomato

Dextran 647
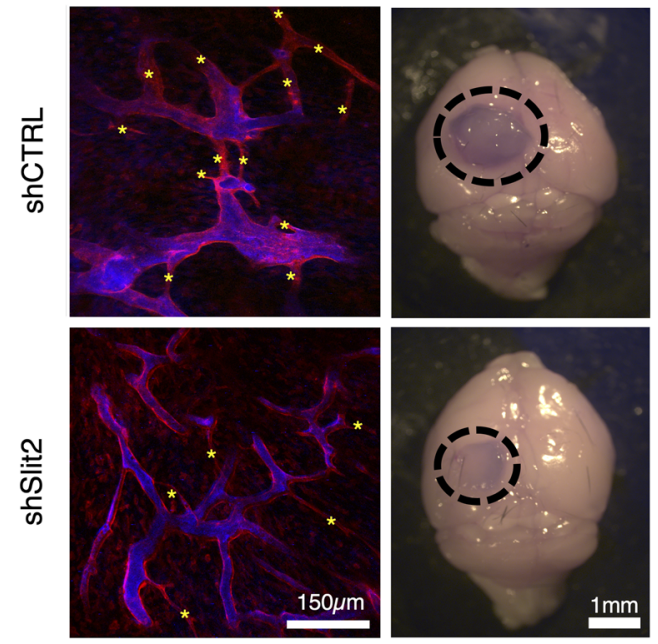

$\mathbf{L}$
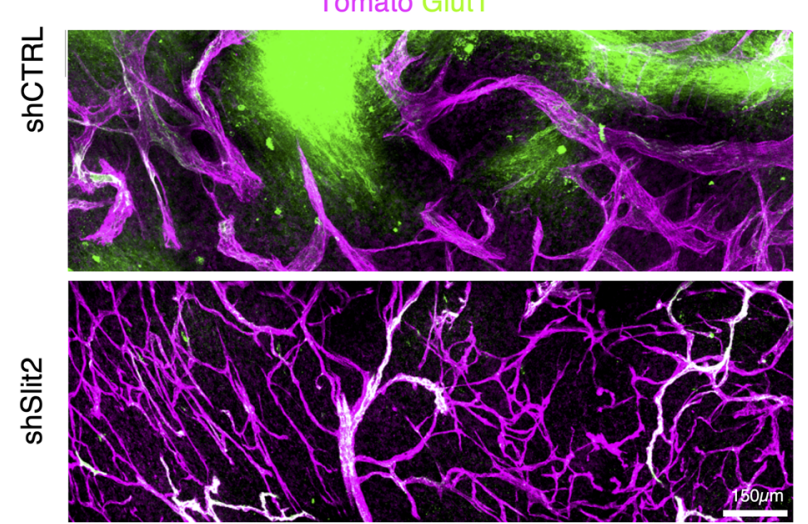
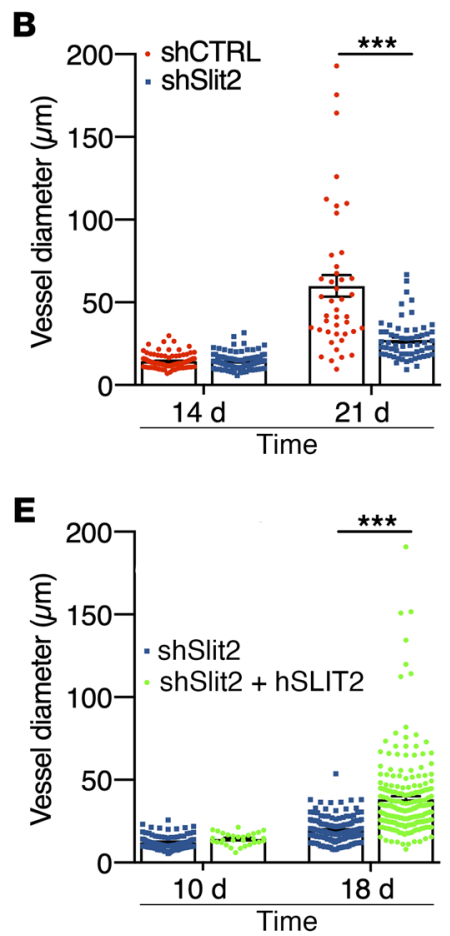

C
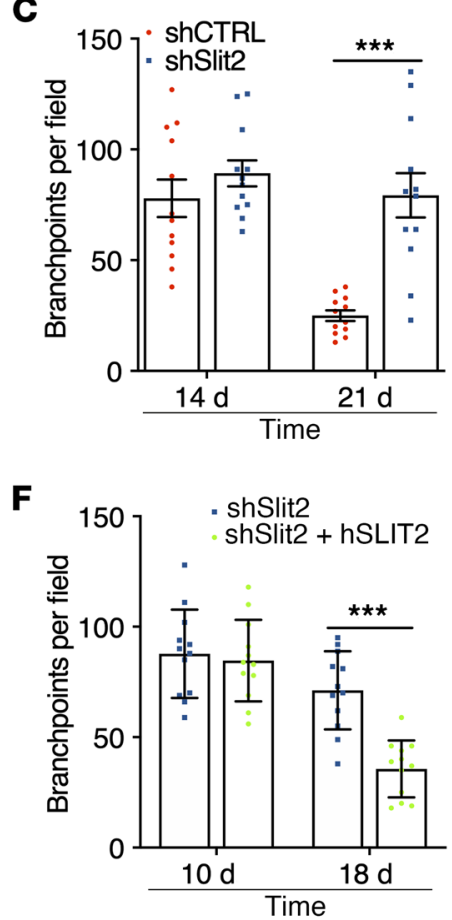

H

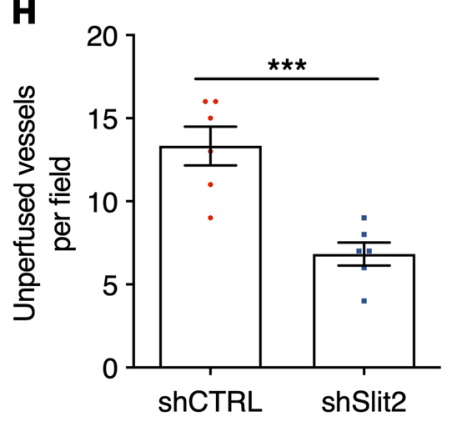

1
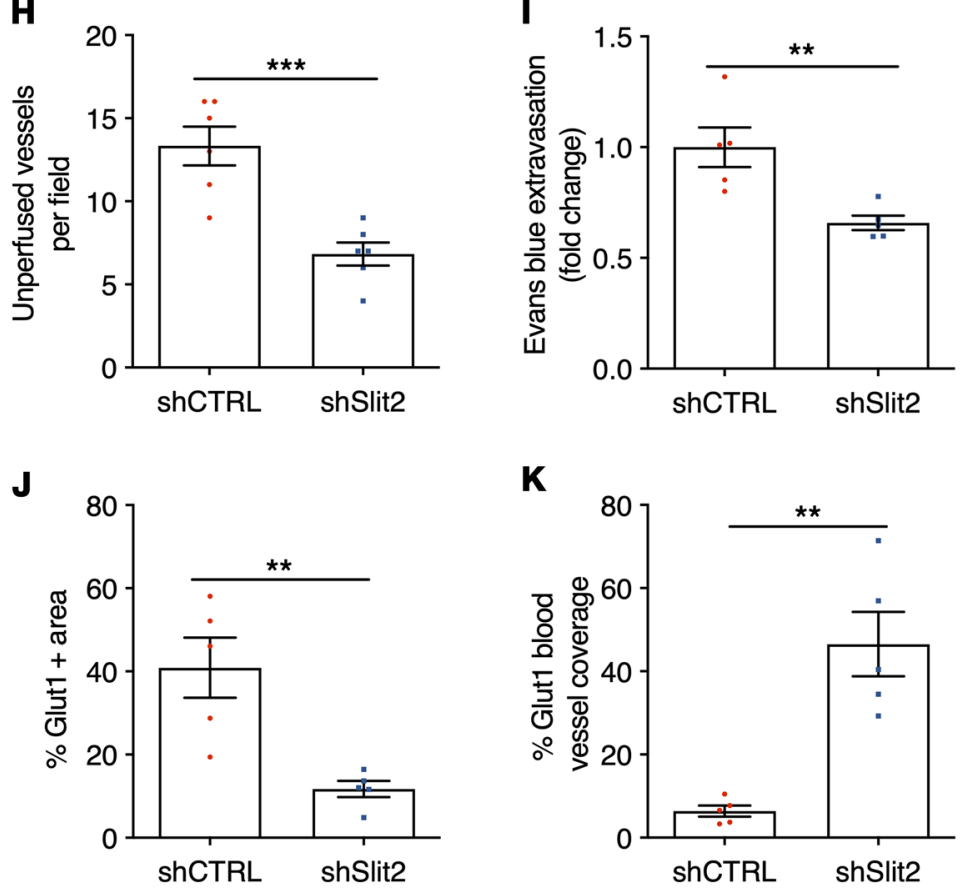
Figure 3. Slit2 promotes blood vessel dysmorphia in GBM. (A) In vivo 2-photon images of ROSA ${ }^{\mathrm{TTm}}$ mice bearing day 21 CT-2A shCTRL or shSlit2 tumors. (B and C) Quantification of vessel diameter (B) and branchpoints (C) ( $n=8$ mice per group, 1-way ANOVA). (D) In vivo 2-photon images of ROSA ${ }^{\text {TTm }}$ mice bearing day 18 CT-2A shSlit2 or shSlit2 + hSLIT2 tumors. (E and F) Quantification of vessel diameter (E) and branchpoints (F) $(n=7$ mice per group, 1-way ANOVA). (G-I) Left: 2-photon in vivo imaging following intravenous injection of Alexa Fluor 647-conjugated Dextran highlighting unperfused blood vessel segments in the tumor core (asterisks) of day 21 CT-2A shCTRL and shSlit2 tumors. Right: representative pictures of whole brains of day 21 shCTRL or shSlit2 CT-2A tumors following Evans blue injection. (H) Quantification of unperfused blood vessel segments in the tumor mass presented in $\mathbf{G}(n=5$ mice per group, Mann-Whitney $U$ test). (I) Quantification of Evans blue extravasation in (G) ( $n=5$ mice per group, Mann-Whitney $U$ test). (J-L) Quantifications of Glut $1^{+}$hypoxic areas in the tumor (J) and Glut1 blood vessel coverage (K) from immunohistochemistry on sections (L) ( $n=5$ mice per group, Mann-Whitney $U$ test). (M) qPCR analyses from FACS-sorted ECs ( $n=3$ tumors/group, Mann-Whitney $U$ test). Data are mean \pm SEM. ${ }^{*} P<0.05,{ }^{* *} P<0.01,{ }^{* * *} P<0.001$.

injection (Figure 3, D-F and Supplemental Figure 5E), just prior to death at 20 days after tumor implantation.

Functionally, in vivo imaging after intravenous Alexa Fluor 647-labeled dextran injection revealed significantly improved perfusion in shSlit2 CT2A tumor vessels when compared with shCTRL tumors (Figure 3, G and H). Quantification of Evans blue extravasation showed reduced vascular leakage in shSlit2 tumors compared with shCTRL (Figure 3I). Along with improved vascular function in shSlit2 knockdown tumors, glucose transporter 1 immunostaining-positive (Glut1-positive) hypoxic areas within the tumor mass were reduced, and Glut1 coverage of blood vessels was increased in shSlit2 knockdown tumors compared with shCTRL, indicating partially improved blood-brain barrier function (Figure 3, J-L). qPCR analysis of sorted tumor endothelial cells $\left(\mathrm{CD} 45^{-} \mathrm{CD} 31^{+}\right)$showed downregulation of immunosuppressive IL-6, PD-L1, and PD-L2 in Slit2 shRNA-transfected tumors compared with CTRL tumors (Figure 3M).

Slit2 silencing reduced myeloid immunosuppression. In vivo imaging also revealed that immune cell infiltration was increased in SLIT2-overexpressing tumors, and decreased in Slit2-silenced tumors when compared with CTRL tumors (Supplemental Figure 6, A-C). Immunofluorescence analysis of tumor sections showed a decrease in the numbers of $\mathrm{F} 4 / 80^{+}$ myeloid cells in day 21 shSlit 2 tumors compared with day 21 shCTRL or day 18 SLIT2-overexpressing tumors (Figure 4, A and B and Supplemental Figure 6, D and E). Activated MHC$\mathrm{II}^{+}$antigen-presenting cells (APCs) were increased in shSlit2 tumors, and $\mathrm{MRC} 1(\mathrm{CD} 206)^{+}$tumor-supportive infiltrating immune cells were decreased (Figure 4, A and B and Supplemental Figure 6, D and E). FACS-sorted $\mathrm{CD}^{4} 5^{+} \mathrm{CD} 11 \mathrm{~b}^{+} \mathrm{F} 4 / 80^{+} \mathrm{Ly} 6 \mathrm{G}$ TAMs accounted for about $12 \%$ of the total cells in shCTRL tumors, but only $6 \%$ in the Slit 2 knockdown tumors (Figure 4C and Supplemental Figure 6F). Half of the TAMs in shSlit2 CT2A tumors had a cytotoxic activation profile and expressed MHC-II and CD11c, while less than 20\% of TAMs in the shCTRL condition expressed MHCII and CD11c and more than $80 \%$ expressed the tumor supportive marker MRC1 (Figure 4D). shSlit2 tumors also showed increased infiltration of dendritic cells $\left(\mathrm{CD} 45^{+} \mathrm{CD} 11 \mathrm{~b}^{-} \mathrm{CD} 11 \mathrm{c}^{+} \mathrm{MHC}-\mathrm{II}^{+} \mathrm{F} 4 / 80^{-}\right)$and neutrophils
$\left(\mathrm{CD} 45^{+} \mathrm{CD} 11 \mathrm{~b}^{+} \mathrm{Ly} 6 \mathrm{G}^{+}\right)$, which were much less abundant when compared with TAMs (Supplemental Figure 6, G and H).

Molecularly, when compared with FACS-sorted shCTRL, TAMs from shSlit2 tumors exhibited decreased expression of the tumor-supportive genes Mrc1, Vegfa, TgfB1, Mmp9, Cd209a, Ccl19, Arg1, and Il10, increased expression of cytotoxic genes Il-12, Il-1b, Ccr7, Cxcl10, and Tnfa, and reduced expression of $P d-l 1$ and $P d-l 2$ inhibitors of $\mathrm{T}$ cell activation (Figure 4E). ELISA analysis showed increased IFN- $\gamma$ and confirmed reduced IL-10 and VEGFa protein levels in TAMs sorted from shSlit2 tumors when compared with controls (Figure 4, F-H). In line with reduced VEGFa expression, in vivo binding of soluble VEGFR1 (sFlt1) showed that only about $40 \%$ of stromal cells in shSlit2 tumors bound sFlt1, while more than $80 \%$ of CTRL and SLIT2overexpressing cells bound Flt1 (Figure 4I).

SLIT2 inhibition increased $T$ cell infiltration and improved $T$ cell-based immunotherapy efficacy. In contrast to the decreased number of TAMs in shSlit2 tumors, the total number of TALs was increased 3-fold (Figure 5A and Supplemental Figure 7, A-D), with an increase in both $\mathrm{CD}^{+}$and $\mathrm{CD} 8^{+} \mathrm{T}$ lymphocytes within the tumor mass when compared with controls (Figure 5, B and $\mathrm{C}$ and Supplemental Figure 7, E-I). Furthermore, the CD $4^{+}$TALs in shSlit2 tumors showed increased expression of Th1 responserelated genes Ifn $\gamma, C x c l 11$, and Il-2, and of IL-17a, but decreased expression of Th2 response-related genes $\mathrm{Il}-1 \mathrm{O}$ and $\mathrm{Cxcl1O}$, and PD-1 and CTLA4 (Figure 5D). CD8 ${ }^{+}$TALs in shSlit2 tumors also showed increased expression of activation markers (IFN- $\gamma$ and GZMB), and reduced expression of genes related to $\mathrm{CD} 8^{+} \mathrm{T}$ cell exhaustion (Tim3 and Lag3; ref. 63 and Figure 5E). In tumor sections, we observed more infiltrating $\mathrm{GZMB}^{+}$activated antitumor $\mathrm{CD}^{+} \mathrm{T}$ cells in shSlit2 compared with shCTRL tumors (Figure $5, F-G)$. ELISA analysis of these sorted CD8 ${ }^{+}$TALs also showed increased IFN- $\gamma$ (Figure 5H) and reduced IL-10 and VEGFa protein levels (Figure 5, I and J) in cells sorted from shSlit2 tumors.

Given this shift toward a less immunosuppressive GBM microenvironment, we hypothesized that shSlit2 tumors could respond to $\mathrm{T}$ cell-based immunotherapy. We tested this idea by treating tumors with anti-PD-1 immune checkpoint inhibitor and agonistic anti-4-1BB antibodies $(11,60)$. We treated mice with $0.2 \mathrm{mg}$ of each antibody at D7, D9, D11, and D13 after tumor implantation. Combining immunotherapy with Slit2 silencing led to powerful antitumor responses, with $100 \%$ of the mice alive at 90 days after implantation (Figure 5K, OS 25 days for shCTRL, 33 days for shCTRL + anti-PD-1 + anti-4-1BB, 33 days for shSlit2 and undetermined for shSlit2 + anti-PD-1 + anti-4-1BB).

The changes in the immune cell microenvironment that we observed in the murine GBM models are also likely to occur in patients with GBM, as shown by positive correlation between SLIT2 and MRC1 and VEGFA mRNA expression in patient samples from our GBM patient cohort and TCGA database cohorts (Supplemental Figure 8, A-C). SLIT2 expression also correlated with genes related to tumor-supportive macrophages (CCL19, CD209, $M M P 9$, and $P D-L 2)$, inhibition of antitumor $\mathrm{T}$ cell responses $(P D-$ 1, CTLA4, CCL17, CXCL11, LAG3, and TIM3), and IL-6 (Supplemental Figure 8, D-O).

SLIT2 promoted microglia and macrophage migration and polarization via $\mathrm{ROBO1}$ and $\mathrm{ROBO} 2$. To determine how SLIT2 affected 
A

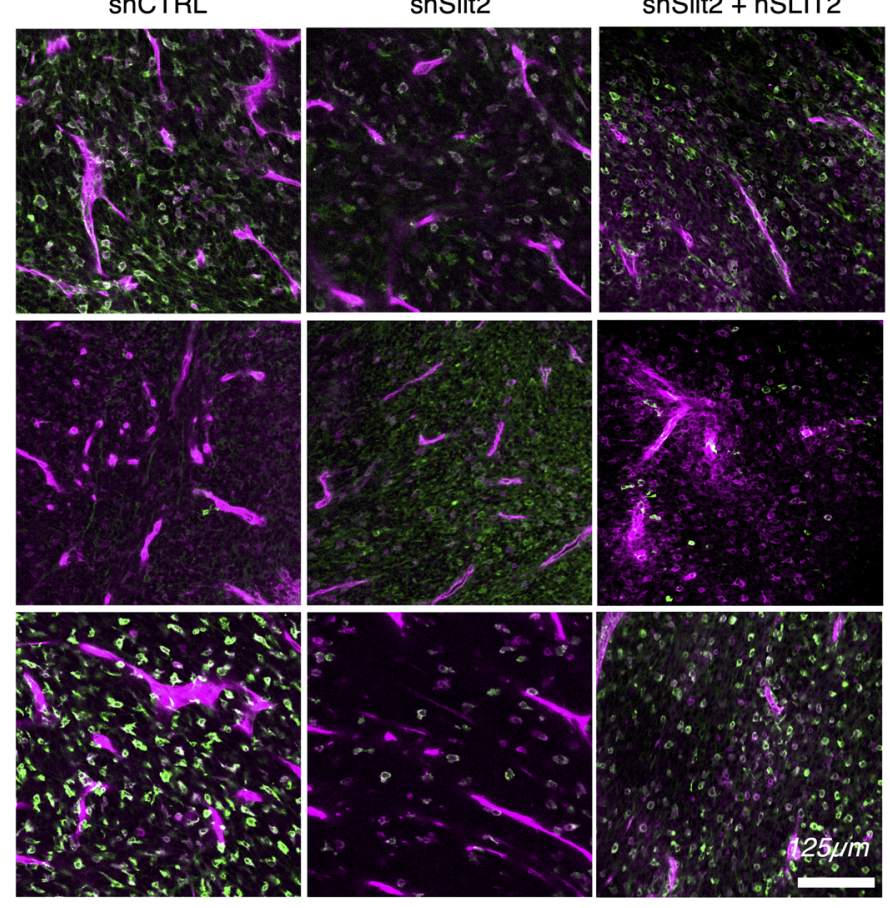

E

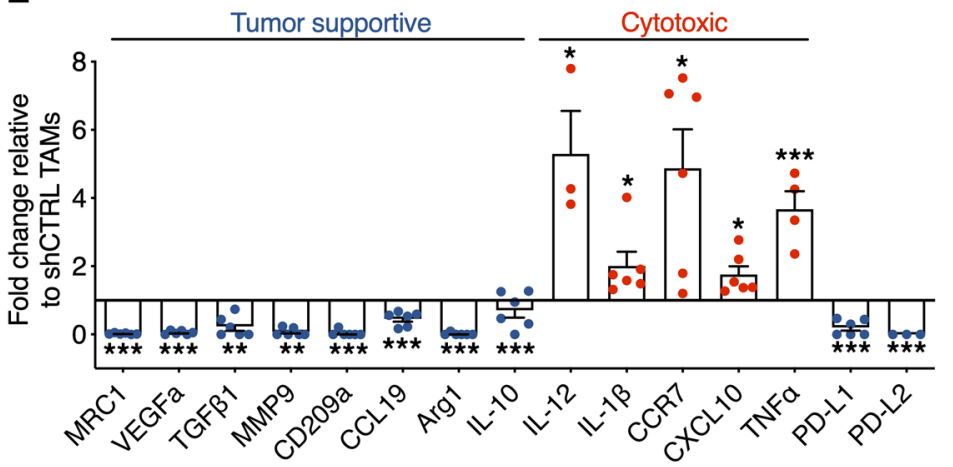

B

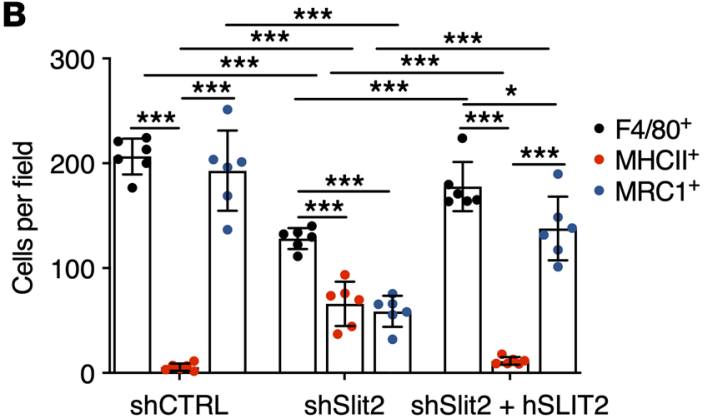

C

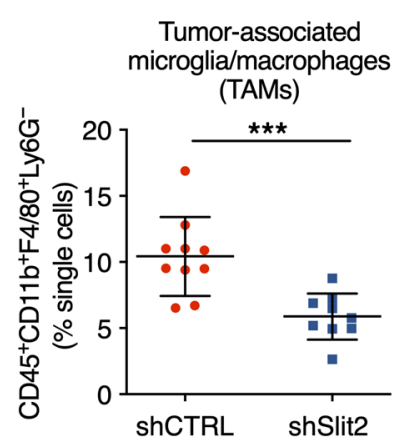

D - Tumor-supportive

TAMs $\left(\mathrm{MRC}^{+}{ }^{+}\right)$

- Cytotoxic TAMs

$\left(\mathrm{MHCll}^{+} \mathrm{CD} 11 \mathrm{C}^{+}\right)$

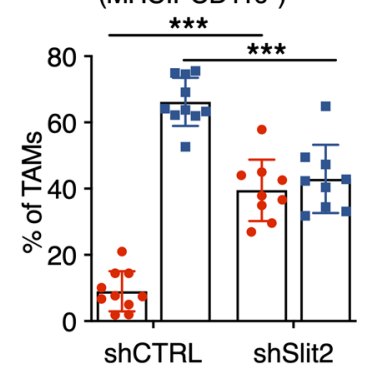

$\mathbf{F}$

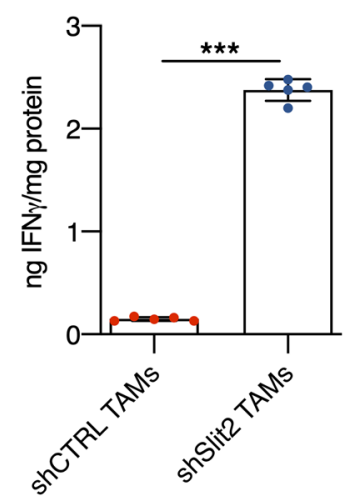

G

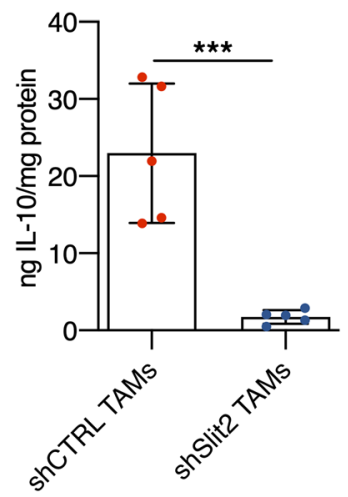

H

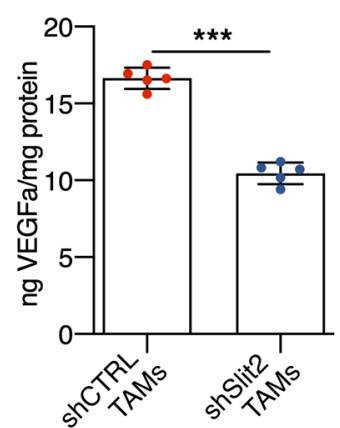

I

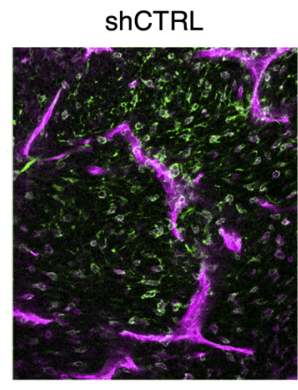

shSlit2

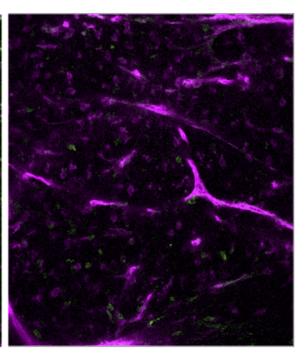

shSlit2 + hSLIT2

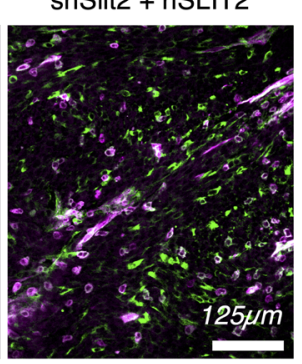

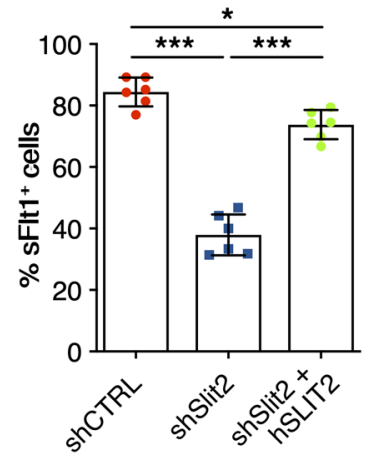

Figure 4. Slit2 promotes TAM recruitment and polarization in mouse gliomas. (A) Immunohistochemistry on sections of late-stage CT-2A shCTRL, shSlit2, or shSlit2 + hSLIT2 tumors for F4/80, MHC-II, and MRC1+ cells (green). (B) Quantifications of $\mathbf{A}$ ( $n=7$ mice per group, 5 fields per tumor, 2 -way ANOVA). (C and D) FACS analysis of day 21 CT-2A shCTRL and shSlit2 tumors for quantification of TAMs ( $n=10$ tumors/group; Student's $t$ test and 2-way ANOVA). (E) qPCR analysis from FACS-sorted TAMs ( $n=6$ tumors/group, Mann-Whitney $U$ test). (F-H) ELISA from protein samples extracted from FACS-sorted TAMs from shCTRL and shSlit2 tumors to quantify IFN- $\gamma(\mathbf{F})$, IL-10 (G), and VEGFa (H) ( $n=5$ tumors/group, Mann-Whitney $U$ test). (I) Representative images and quantification of soluble-Flt1 binding to sections of day 21 CT-2A shCTRL, shSlit2, and day 18 shSlit2 + hSLIT2 tumors ( $n=7$ mice per group, 5 fields per tumor, 1-way ANOVA). ${ }^{*} P<0.05,{ }^{* *} P<0.01,{ }^{* * *} P<0.001$. 
A

Tumor-associated T lymphocytes (TALs)

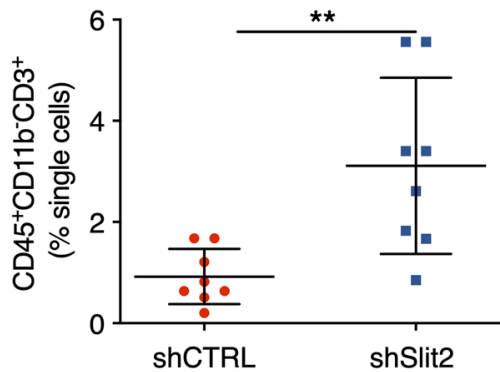

B

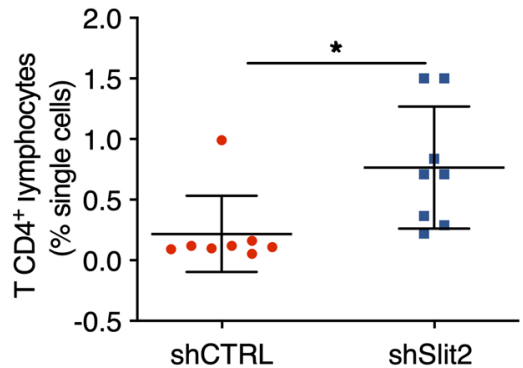

C

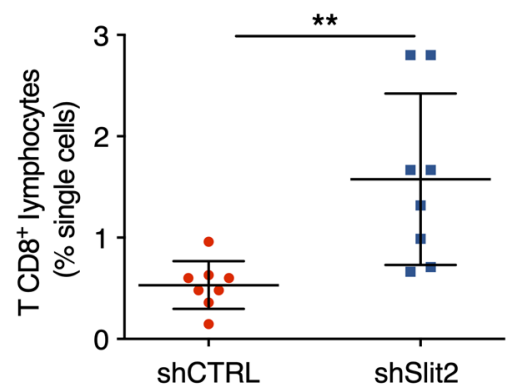

D

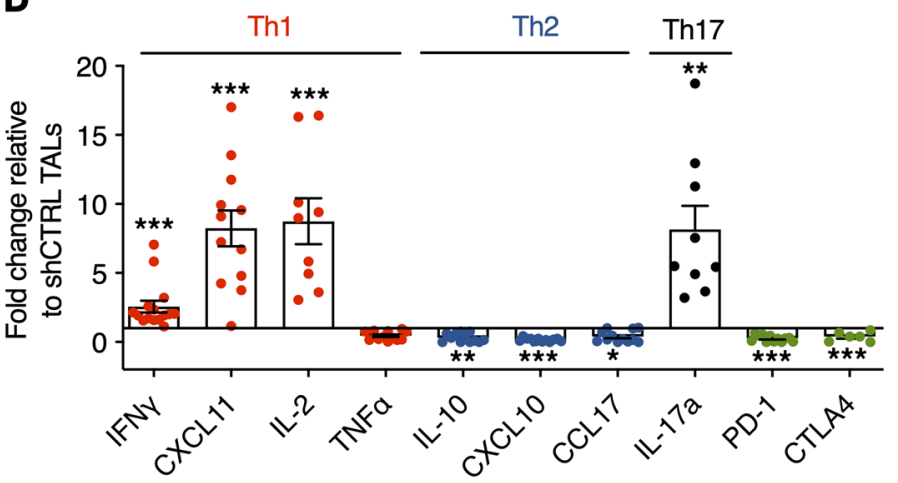

E

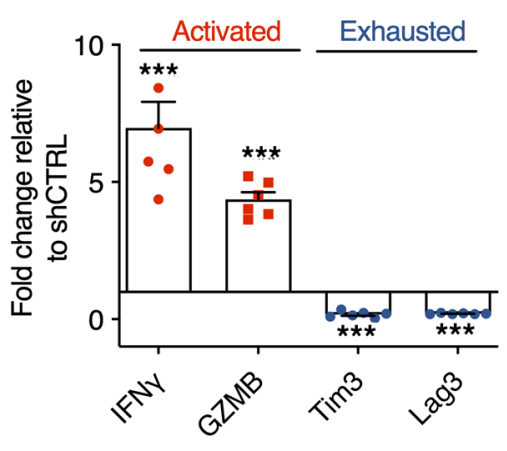

$\mathbf{F}$

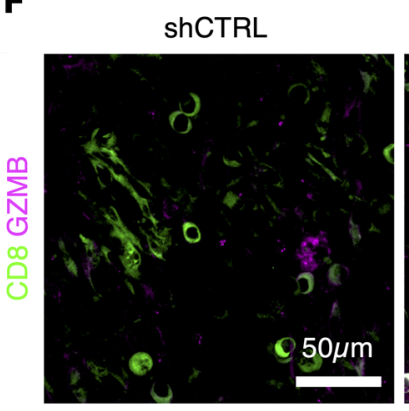

I

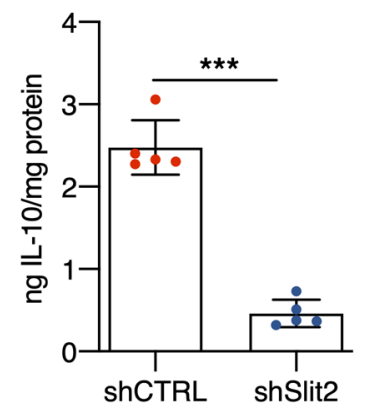

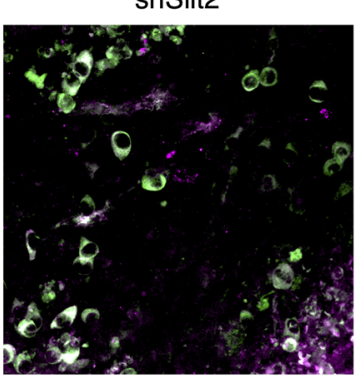

$\mathbf{J}$

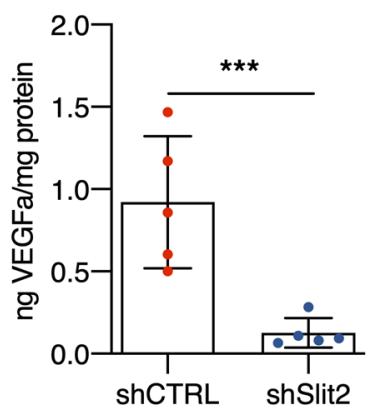

G

H
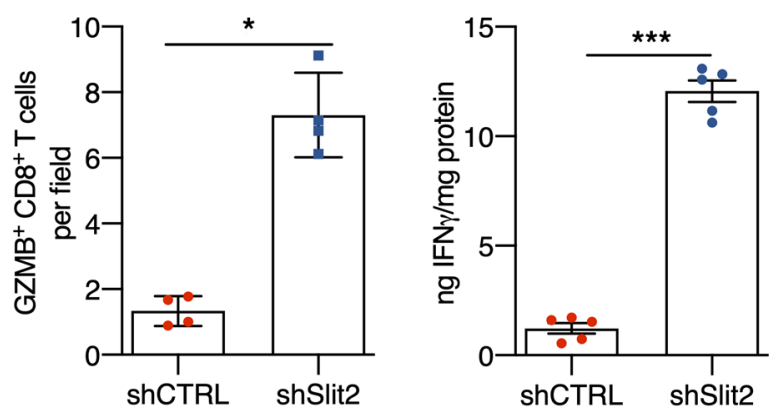

$\mathbf{K}$

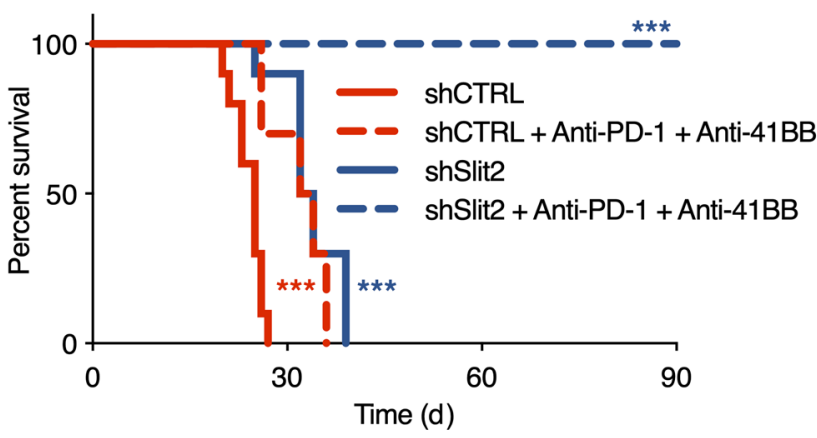

Figure 5. Slit2 inhibits T lymphocyte responses in the glioma microenvironment. (A-C) T lymphocyte FACS analysis of day 21 CT-2A shCTRL and shSlit2 tumors for total CD3 $3^{+}$TALs (A), CD4 $4^{+}$TALs (B), and CD8 $8^{+}$TALs (C) ( $n=8$ tumors/group; Student's $t$ test). (D) qPCR analyses from FACS-sorted CD4 ${ }^{+} T$ lymphocytes ( $n=10$ tumors/group, Mann-Whitney $U$ test). (E) qPCR analyses from FACS-sorted CD8 ${ }^{+}$T lymphocytes ( $n=6$ tumors/group, Mann-Whitney $U$ test). (F) Representative images of CD8 and CZMB staining on sections of day 21 CT-2A shCTRL and shSlit2 tumors. (G) Quantification of F ( $n=4$ mice per group, 5 fields per tumor, Mann-Whitney $U$ test). (H-J) ELISA from protein samples extracted from FACS-sorted CD8 ${ }^{+}$TALs from shCTRL and shSlit2 tumors to quantify IFN- $\gamma(\mathbf{H})$, IL-10 (I), and VEGFa (J) ( $n=5$ tumors/group, Mann-Whitney $U$ test). (K) Eight-week-old mice were engrafted with CT-2A shCTRL or shSlit2 and randomly assigned to vehicle or anti-PD-1 + anti-4-1BB treatment (0.2 mg each on days 7, 9, 11, and 13 after tumor implantation) ( $n=10 / 11$ mice per group, OS 25 days for shCTRL, 33 days for shCTRL + anti-PD-1 + anti-4-1BB, 33 days for shSlit2, and undetermined for shSlit2 + anti$\mathrm{PD}-1+$ anti-4-1BB; multiple comparisons log-rank test). Data are mean $\pm \mathrm{SEM} .{ }^{*} P<0.05,{ }^{* *} P<0.01,{ }^{* *} P<0.001$. 

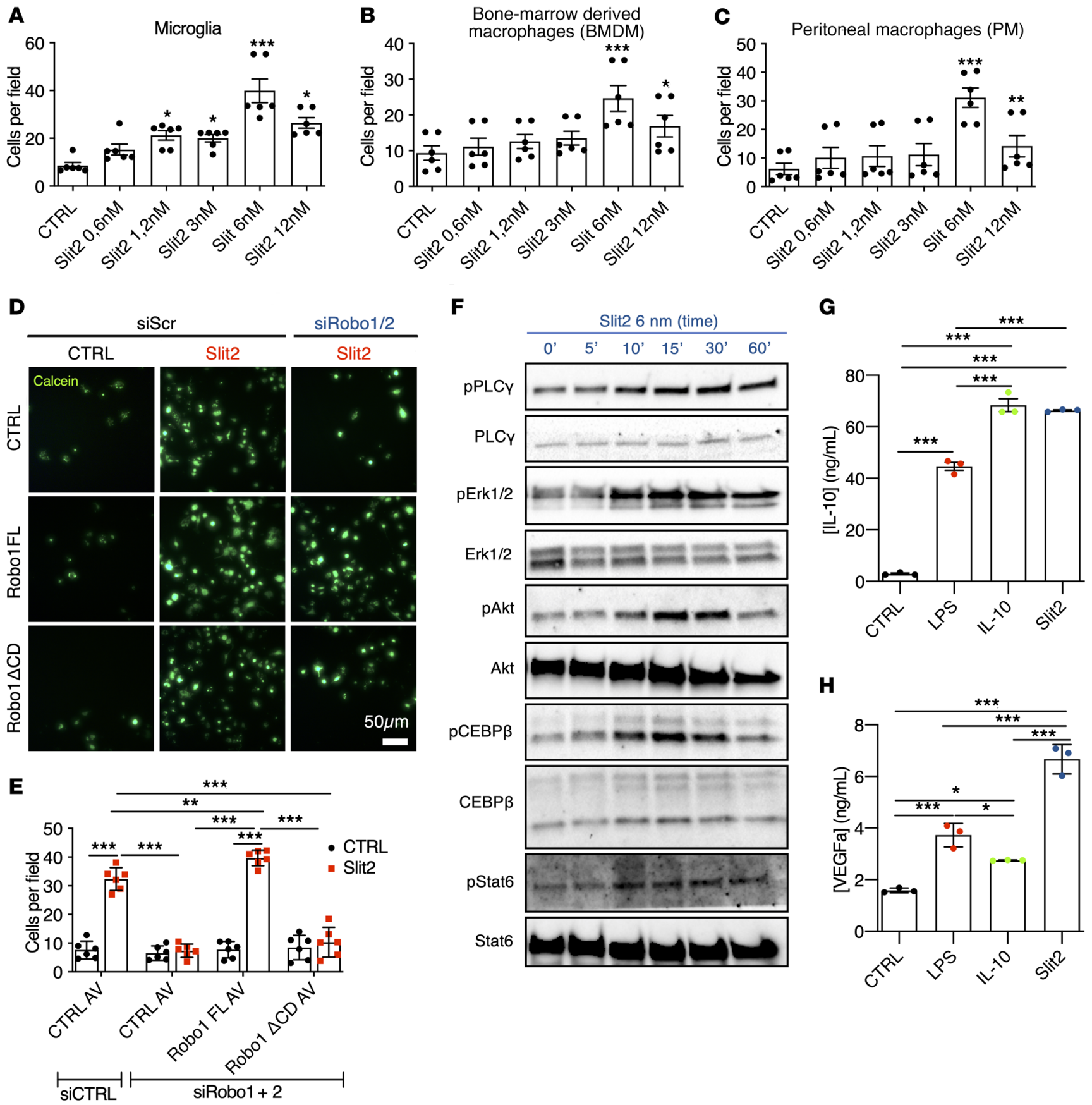

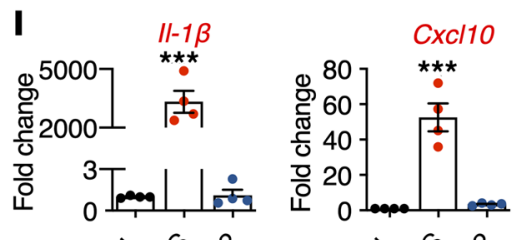

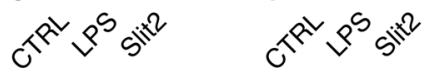
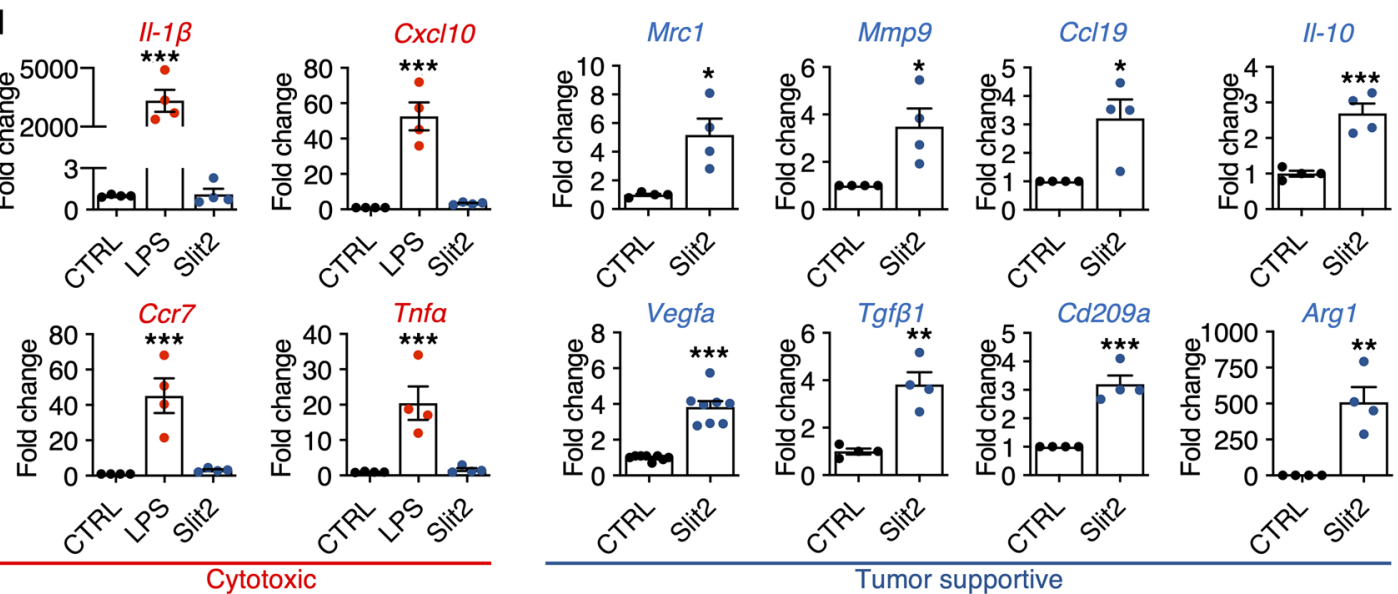

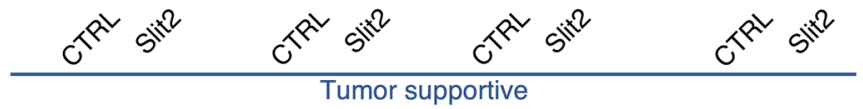


Figure 6. Slit2 drives microglia and macrophage migration and tumor supportive polarization. (A-C) Transwell assay of microglial cells (A), BMDMs (B), and PMs (C) in response to Slit2 or carrier (CTRL) in the bottom chamber ( $n=4$, 1-way ANOVA). ( $\mathbf{D}$ and $\mathbf{E})$ Transwell assay of RAW macrophages treated or not with Robo1/2 siRNA and infected with adenovirus encoding CTRL (GFP construct), Robo1FL, or Robo1 $\triangle$ CD constructs and stained with calcein. (E) Quantification of $\mathbf{D}(n=3,2$-way ANOVA). (F) Western blot analysis of Slit2 downstream signaling in cultured BMDMs $(n=6)$. (G and $\mathbf{H})$ ELISA from conditioned medium from LPS-, II-10-, or Slit2-treated BMDMs to quantify IL-10 (C) and VEGFa (H) ( $n=3$ independent cultures, Mann-Whitney $U$ test). (I) qPCR analysis of BMDM cultures following Slit2 or LPS treatment ( $n=4,1$-way ANOVA or Mann-Whitney $U$ test). Data are mean \pm SEM. ${ }^{*} P<0.05,{ }^{* *} P<0.01,{ }^{* *} P<0.001$.

myeloid cells, we tested microglia and macrophage migration in Transwell chambers. Slit2 in the bottom chamber induced chemotaxis of isolated mouse microglial cells, bone marrow-derived macrophages (BMDMs) and peritoneal macrophages (PMs) in a dose-dependent manner, with a maximum response at $6 \mathrm{nM}$ (Figure $\left.6, \mathrm{~A}^{-} \mathrm{C}\right)$. Adding Slit2 to both top and bottom chambers inhibited macrophage migration, indicating a chemotactic response (Supplemental Figure 9, A and B).

To determine if SLIT2 signaled through ROBO receptors to promote macrophage migration, we silenced Robo1 and Robo2 in cultured RAW264.7 macrophages using siRNAs, which inhibited Slit2-induced macrophage migration (Figure 6, D and E and Supplemental Figure 9, C and D). Migration could be rescued by adenoviral-induced expression of a siRNAresistant full-length rat Robo1 construct (Robo1FL) but not by a construct lacking the cytoplasmic signaling domain (Robo1 $\triangle \mathrm{CD}$; Figure 6, D and E).

To identify SLIT2 downstream signaling pathways in macrophages, we treated BMDM and microglial cells with $6 \mathrm{nM}$ Slit2, which led to PLC- $\gamma$, Erk1/2, and Akt phosphorylation (Figure 6F and Supplemental Figure 9, E-G, and J). SLIT2 also induced phosphorylation of Stat6 and CEBP $\beta 1$, which polarize tumorinfiltrating macrophages toward a tumor supportive phenotype (ref. 64, Figure 6F, and Supplemental Figure 9, H-J), suggesting that SLIT2 induced tumor-supportive gene expression changes.

Conditioned medium of Slit2-treated microglia and macrophages had increased levels of IL-10 and VEGFa compared with cells not treated with Slit2 (Figure 6, G and $\mathrm{H}$ and Supplemental Figure 9, K and L). The expression of genes characteristic of a tumor supportive macrophage phenotype, including Mrcl, Vegfa, Mmp9, TgfB1, Ccl19, Cd209a, Il-10, and Arg1, were all increased by Slit2 treatment, while cytotoxic response-related genes $I l-1 \beta$, Cxcl10, Ccr7, and Tnfa were unaffected by Slit2 but increased by LPS (Figure 6I). Slit2-induced gene expression changes were ROBO1 and ROBO2 dependent, as shown by siRNA silencing of Robo1/2, which abrogated Slit2 induced changes in protein phosphorylation and gene expression (Supplemental Figure 10, A-G).

SLIT2/ROBO induced tumor-supportive macrophage/microglia polarization via PI3K- $\gamma$. Previous studies have shown that Stat6 and CEBP $\beta 1$ activation in TAMs occur downstream of PI3K- $\gamma(64)$, leading us to ask if SLIT2/ROBO1 and ROBO2 signaled upstream of PI3K- $\gamma$ to induce macrophage tumor-supportive polarization. First, we observed Robo1 and PI3K- $\gamma$ coimmunoprecipitation in BMDMs, which was enhanced after Slit2 treatment for 15 minutes
(Figure 7A). Second, Slit2-induced BMDM migration was abrogated by pretreatment with a specific PI3K- $\gamma$ inhibitor IPI-549 $(1 \mu \mathrm{M}$, Figure $7 \mathrm{~B})$. Third, Slit2-induced phospho-Stat6 nuclear translocation in cultured BMDMs was prevented by pretreatment with IPI-549 (Figure 7, C and D). Slit2-induced Akt and Stat6 phosphorylation (Supplemental Figure 11A), as well as IL-10 and VEGFa secretion in ELISA from BMDM-conditioned medium were also reduced by PI3K- $\gamma$ inhibition (Figure 7, E and F). Finally, the Slit2-induced expression of genes characteristic of a tumor supportive macrophage phenotype (Mrc1, Vegfa, Mmp9, Tgf $\beta 1$, Ccl19, Cd209a, Il-10, and Arg1) was disrupted by IPI-549 pretreatment, while LPS-induced cytotoxic response-related genes were unaffected in both BMDMs and microglial cells by PI3K- $\gamma$ inhibition (Figure $7 \mathrm{G}$ and Supplemental Figure 11B).

Robo deficiency in TAMs inhibited glioma growth and vascular dysmorphia. To determine if SLIT2 signaling effects in macrophages were sufficient to drive the stromal response, we developed mice with genetic Robo receptor deletions in macrophages. To do so, we intercrossed Robo1 ${ }^{-/}$Robo $2^{\mathrm{n} / \mathrm{l}}$ mice (28) with CSF-1R ${ }^{\text {CreeRT2 }}$ mice (65) on a ROSA ${ }^{\mathrm{mT} / \mathrm{mG}}$ background, generating Robo1 ${ }^{-/}$Robo $2^{\mathrm{t} / \mathrm{f}} \mathrm{CSF}-1 \mathrm{R}^{\mathrm{CreERT} 2} \mathrm{ROSA}^{\mathrm{mT} / \mathrm{mG}}$ mice (hereafter named iRoboMacKO mice). Littermate Robo1 $^{+/-}$Robo2 $^{+/ f l}$ CSF-1 ${ }^{\text {CreERT2}} \mathrm{ROSA}^{\mathrm{mT} / \mathrm{mG}}$ or Robo1 ${ }^{-/}$Robo $2^{\mathrm{f} / \mathrm{l} \mathrm{R}} \mathrm{ROSA}^{\mathrm{mT} / \mathrm{mG}}$ mice were used as controls. Mice were implanted with CT-2A-BFP glioma cells and followed longitudinally during tumor growth. Tamoxifen injections to induce gene deletion were done every 3 days starting 7 days after tumor implantation, and induced robust gene deletion, assessed by qPCR of $\mathrm{GFP}^{+}$cells extracted from the bone marrow of tumor-bearing mice (Supplemental Figure 12, A and B).

MRI imaging and histological analysis 21 days after tumor implantation converged to show reduced tumor size in iRoboMacKO tumors when compared with controls (Figure 8, $\mathrm{A}-\mathrm{C})$. T1-weighted imaging after gadolinium injection showed a more homogeneous contrast signal in iRoboMacKO tumors, while control GBMs displayed predominantly peripheral and heterogenous contrast distribution, suggesting improved perfusion in iRoboMacKO tumors (Figure 8A). In vivo 2-photon imaging revealed that blood vessels in iRoboMacKO tumors dilated less and remained more ramified when compared with controls (Figure $8, \mathrm{D}-\mathrm{F})$. Glut $1^{+}$hypoxic zones within the tumor mass were reduced in iRoboMacKO tumors, confirming improved perfusion when compared with controls (Figure 8, G and H). Most of the Glut1 staining in iRoboMacKO tumors colocalized with Tomato $^{+}$blood vessels, attesting to the qualitative improvement of iRoboMacKO tumor vessels (Figure 8G).

Compared with controls, iRoboMacKO tumors displayed reduced overall numbers of intratumor $\mathrm{Iba}^{+}$myeloid cells, with a significant increase of cytotoxic $\mathrm{MHCII}^{+}$cells and a reduction in tumor-supportive $\mathrm{MRC1}^{+}$cells (Figure 8I and Supplemental Figure 12D). Soluble Flt1 binding was reduced in iRoboMacKO tumors (Figure 8J and Supplemental Figure 12D), and Robo1/2-deleted cells extracted from the bone marrow of tumor-bearing mice showed decreased Vegfa expression (Supplemental Figure 12C).

$\mathrm{T}$ cell infiltration was increased in iRoboMacKO tumors (Figure $8 \mathrm{~K}$ and Supplemental Figure 12D), suggesting that SLIT/ROBO signaling inhibition in macrophages was suffi- 
A

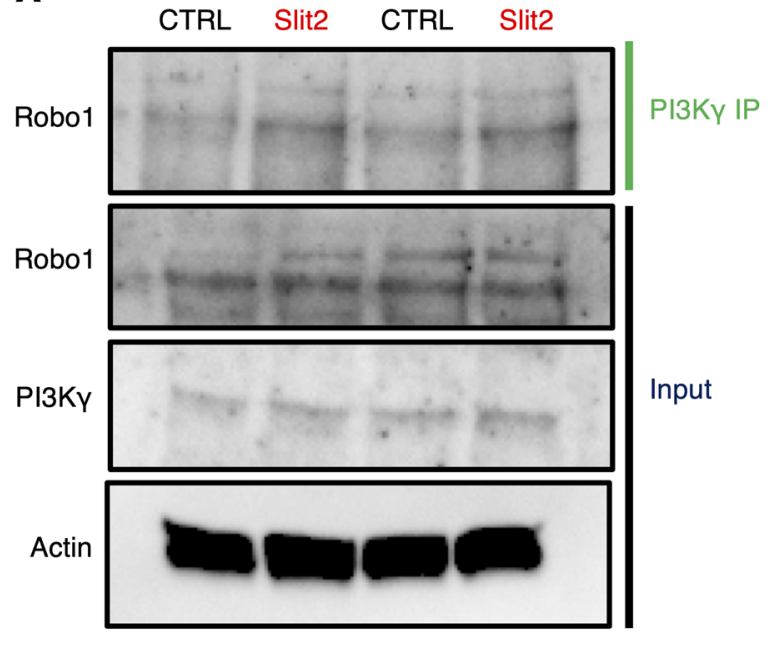

B

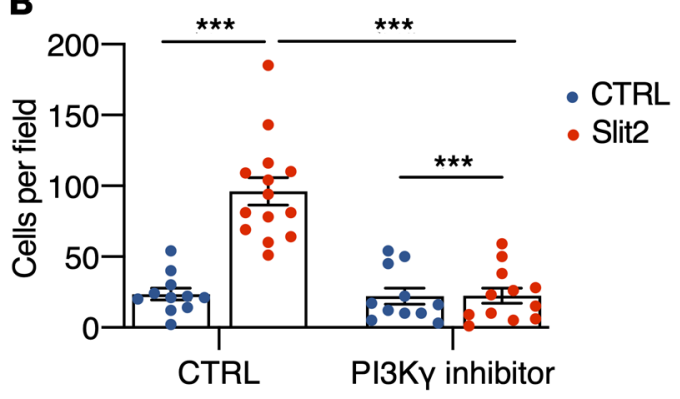

$\mathbf{E}$

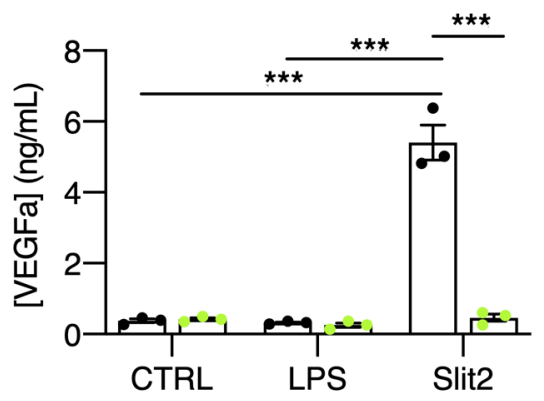

G

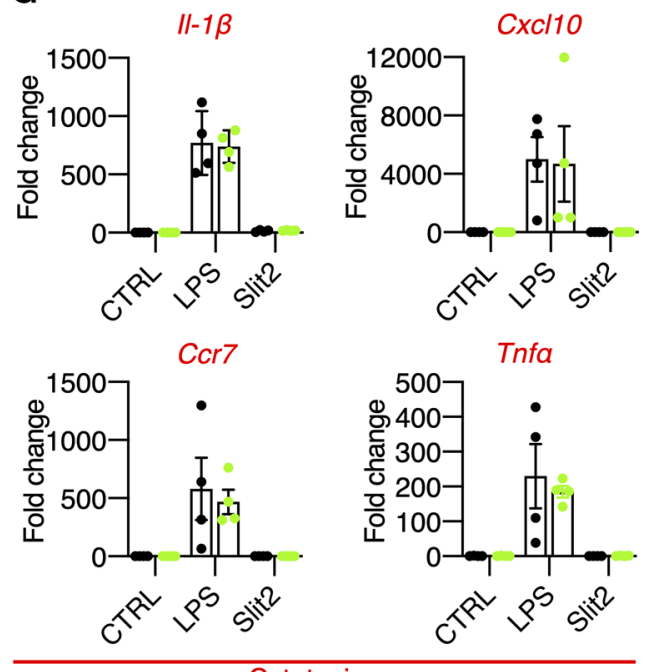

Cytotoxic
C
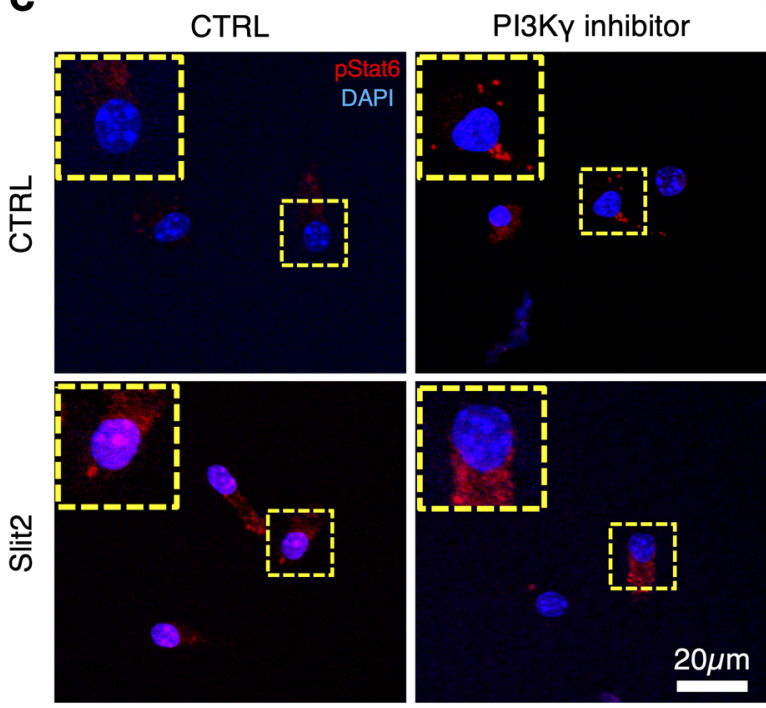

D

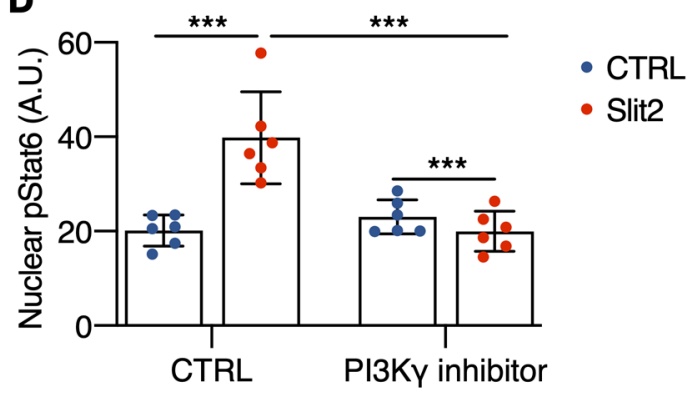

$\mathbf{F}$

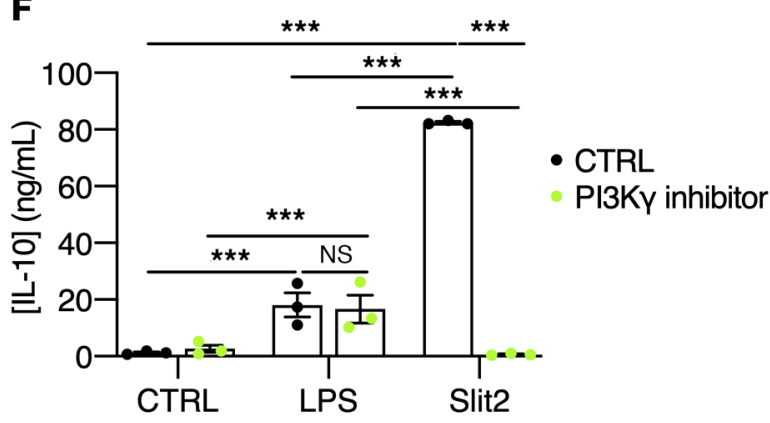

- CTRL

PI3Ky inhibitor

CTRL

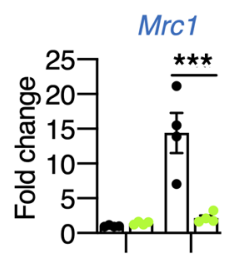

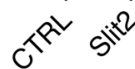

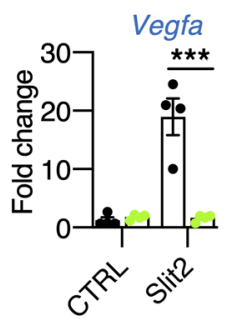

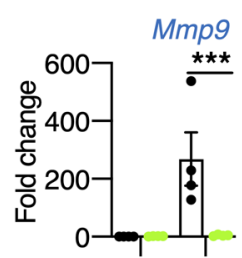
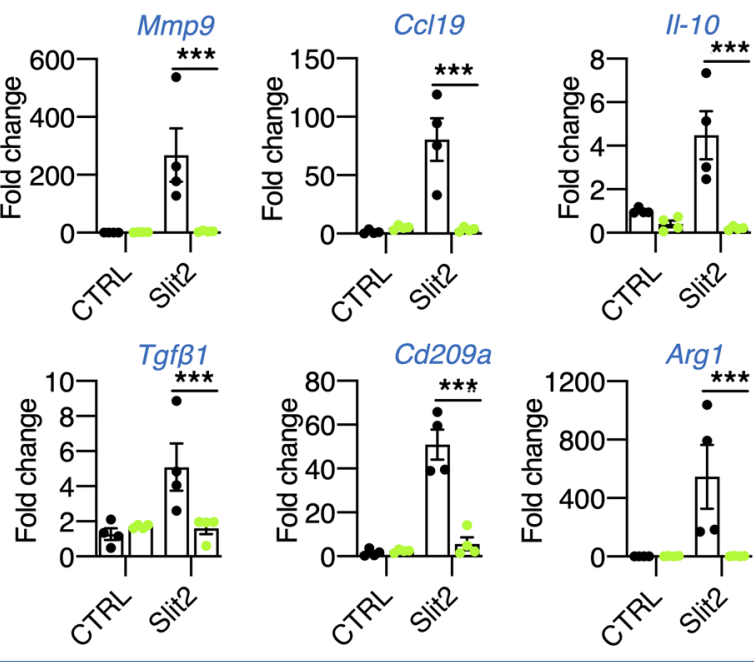
Figure 7. Slit2-driven microglia/macrophage polarization via PI3K- $\gamma$. (A) PI3K- $\gamma$ immunoprecipitation in BMDMs treated or not with Slit2 for 15 minutes and Western blot for Robo1 ( $n=3$ independent experiments). (B) Transwell assay of BMDMs in response to Slit2 or carrier (CTRL) in the bottom chamber after pretreatment with vehicle control (DMSO) or PI3K- $\gamma$ inhibitor IPI-549 (1 $\mu \mathrm{M})$. (C and D) Phospho-Stat6 immunofluorescent staining of BMDMs treated or not with Slit2 and PI3K- $\gamma$ inhibitor and quantification of nuclear pStat6 intensity ( $n=4$ independent cultures, 2-way ANOVA). (E and F) ELISA from conditioned medium from LPS- or Slit2-treated BMDMs with vehicle control (DMSO) or PI3K- $\gamma$ inhibitor, to quantify IL-10 (E) and VEGFa (F) ( $n=3$ independent cultures, 2-way ANOVA). (C) qPCR analysis of BMDM cultures following Slit2 or LPS treatment with vehicle control or PI3K- $\gamma$ inhibitor ( $n=4$ independent cultures, 2-way ANOVA). Data are mean \pm SEM. ${ }^{*} P<0.05,{ }^{* * *} P<0.001$.

cient to shift the GBM microenvironment toward a cytotoxic, T cell-enriched phenotype. This effect could be due to increased circulation of APCs to the tumor draining lymph-nodes, where they can activate antitumor $\mathrm{T}$ cell responses (60). Analysis of glioma-draining deep cervical and mandibular lymph nodes (DCLNs and MLNs, respectively) for the presence of BFP tumor antigen in immune cells revealed an important increase in CD11 $\mathrm{b}^{+} \mathrm{BFP}^{+}$cells in both DCLNs and MLNs of iRoboMacKO tumors when compared with controls (Figure 8, L and $\mathrm{M}$ and Supplemental Figure 12E).

Lymphocyte sequestration in the bone marrow contributes to the $\mathrm{T}$ cell-depleted TME and failure of currently available immunotherapy (11). iRoboMacKO mice had significantly increased lymphocyte counts in peripheral blood 21 days after tumor implantation (Figure 8N). Given that total WBC count was not changed (Supplemental Figure 12F), tumor-bearing iRoboMacKO mice shifted to a predominance of lymphocytes over neutrophils in the blood stream (Figure 80), revealing a reduction in the systemic immunosuppression after macrophage-specific Robo1 and Robo 2 knockout.

Given the profound changes observed in the TME, we next tested if macrophage-specific Robo1 and Robo2 deletion was sufficient to prolong survival and sensitivity to T cell-based immunotherapy. Indeed, macrophage-specific Robo1/2 knockout increased survival of tumor-bearing mice (Figure 8P, OS 21.5 days for Robo1 ${ }^{-/}$Robo2 $2^{\mathrm{f} / \mathrm{l}}, 29$ days for iRoboMacKO), and survival benefit was further increased by immunotherapy, with 70\% of the iRoboMacKO mice alive after 100 days (Figure 8P, OS 24 days for Robo1 ${ }^{-/}$Robo2 ${ }^{\mathrm{f} / \mathrm{Al}}+$ anti-PD-1 + anti-4-1BB, undefined for iRoboMacKO + anti-PD-1 + anti-4-1BB).

In contrast to macrophage Robo depletion, $\mathrm{T}$ cell depletion using anti-CD3 145-2C11 antibodies (66) did not induce significant changes of blood vessels or TAMs in the GBM microenvironment (Supplemental Figure 13).

Systemic SLIT2 inhibition alleviated GBM immunosuppression. We reasoned that systemic administration of a SLIT2 ligand trap protein (Robo1Fc) might be efficient in a therapeutic setting. Mice with established shCTRL CT2A tumors were intravenously injected 5 times with $2.5 \mathrm{mg} / \mathrm{kg}$ Robo1Fc every second day starting from day 7 after tumor implantation and were analyzed at day 23 (Figure 9A). Control mice received injections of human control IgG1 Fc fragment. Robo1Fc treatment reduced Slit2 serum levels, as attested by Slit2 ELISA on days 14 and 21 after tumor implantation (Figure 9B). Mice treated with Robo1Fc exhibited a pronounced tumor growth reduction compared with control Fc-treated tumors (Figure 9, C and D). MRI analysis 21 days after tumor implantation showed that tumor size was reduced and that tumor perfusion was improved, as seen by the more homogeneous gadolinium uptake in Robo1Fc-treated tumors compared with controls (Supplemental Figure 14, A and B). In vivo imaging demonstrated that Robo1Fc treatment reduced vascular dysmorphia (Figure 9, E-G) and reduced Glut1 ${ }^{+}$hypoxic zones within the tumor mass (Figure $9 \mathrm{H}$ and Supplemental Figure 14C). Robo1Fc treatment changed immune cell infiltration and reduced overall numbers of intratumoral $\mathrm{F} 4 / 80^{+}$cells, with a significant increase of cytotoxic $\mathrm{MHCII}^{+}$cells and a reduction of tumor-supportive $\mathrm{MRC}^{+}{ }^{+}$cells compared with controls (Figure 9I and Supplemental Figure 14D). Soluble Flt1 binding was reduced in Robo1Fc-treated tumors (Figure 9J and Supplemental Figure 14D), while T cell infiltration was increased compared with controls (Figure 9K and Supplemental Figure 14D).

Analysis of glioma-draining DCLNs and MLNs showed an increased presence of GFP tumor antigen in APCs $\left(C D 45^{+} \mathrm{CD}-\right.$ $\left.11 b^{+}{ }^{2} 6 G^{-}\right)$of Robo1Fc-treated mice when compared with CTRLFc-treated ones (Supplemental Figure 14, E-G), as we observed in iRoboMacKO mice. Finally, Robo1Fc-treated mice also had significantly increased total WBC and lymphocyte counts in peripheral blood 21 days after tumor implantation, and we observed a shift to a predominance of lymphocytes over neutrophils in the blood stream of Robo1Fc-treated mice, while other WBC counts were unchanged (Supplemental Figure 14, H-J).

Five injections of Robolfc protein during early stages of tumor progression were sufficient to significantly extend survival of tumor-bearing mice, and $25 \%$ of the treated mice survived 150 days after implantation (Figure 9L, OS 24 days for CTRLFc and 41 days for Robo1Fc). Combining Robo1Fc with TMZ further increased this survival benefit, with $45 \%$ of the mice surviving 150 days after implantation (Figure 9L, OS 28 days for CTRLFc + TMZ and 119 days for Robo1Fc + TMZ). Combining Robo1Fc with anti-PD-1 and anti-4-1BB antibodies further improved antitumor responses, with $80 \%$ of the mice surviving 90 days after tumor implantation (Figure 9M, OS 40.5 days for CTRLFc + anti-PD-1 + anti-4-1BB and undefined for Robo1Fc + anti-PD-1 + anti-4-1BB). Mice that survived the immunotherapy were rechallenged by a second tumor injection in the contralateral hemisphere. Mice that survived after treatment with Robo1Fc and T cell-based immunotherapy had the best long-term survival after tumor rechallenge, with more than $80 \%$ of mice alive 90 days after tumor reinjection (Figure 9N, OS 22 days for naive mice, 53.5 days for anti-PD-1 + anti-4-1BB survivors, 63 days for Robo1Fc survivors, and undefined for Robo1Fc + anti-PD-1 + anti-4-1BB survivors).

\section{Discussion}

Collectively, our data showed that GBM-derived SLIT2 signaled through $\mathrm{ROBO} 1$ and $\mathrm{ROBO} 2$ in TAMs, which resulted in an impairment of antitumor immunity and the induction of vascular dysmorphia in the TME. SLIT2/ROBO1 and ROBO2 signaling is therefore a potentially novel immune evasion mechanism in the TME, and inhibiting this pathway in TAMs could sensitize GBM to T cell-based immunotherapy, and add to the therapeutic arsenal against GBM. 
A

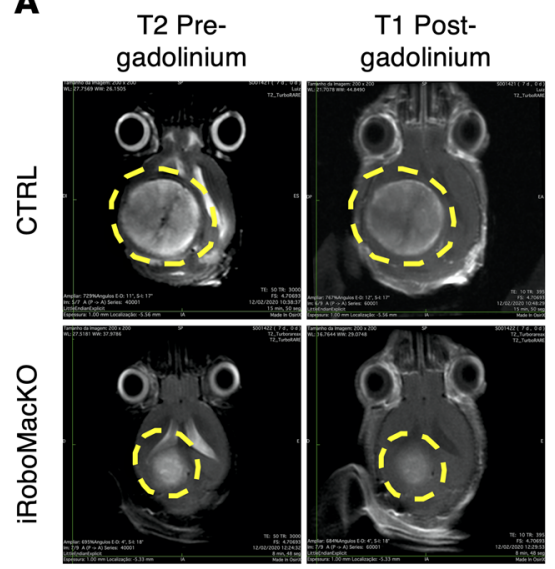

E
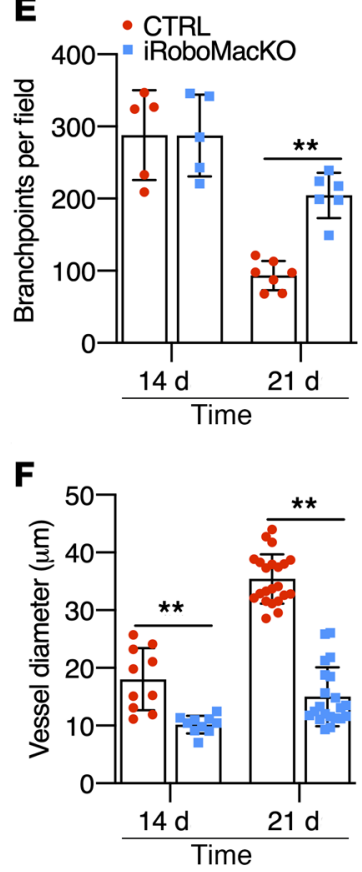

B

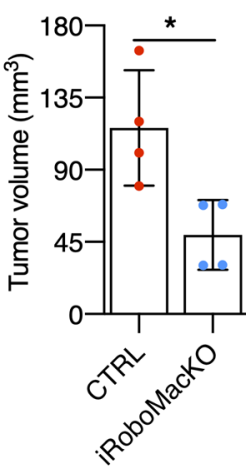

G

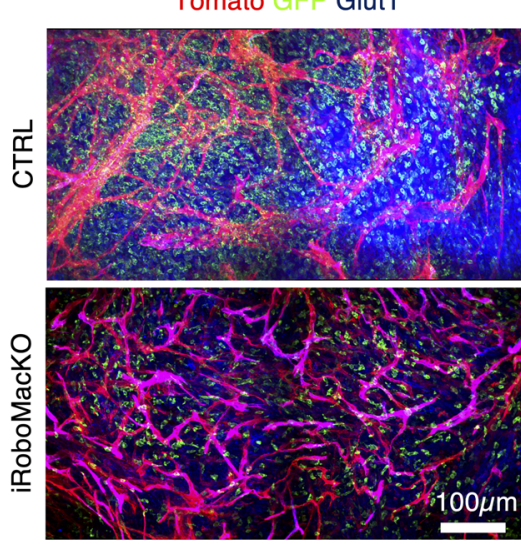

C

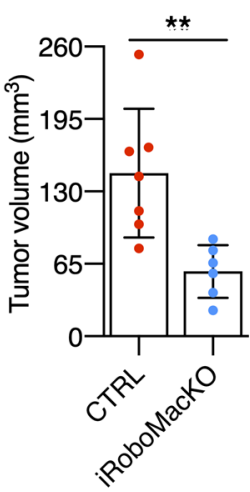

H
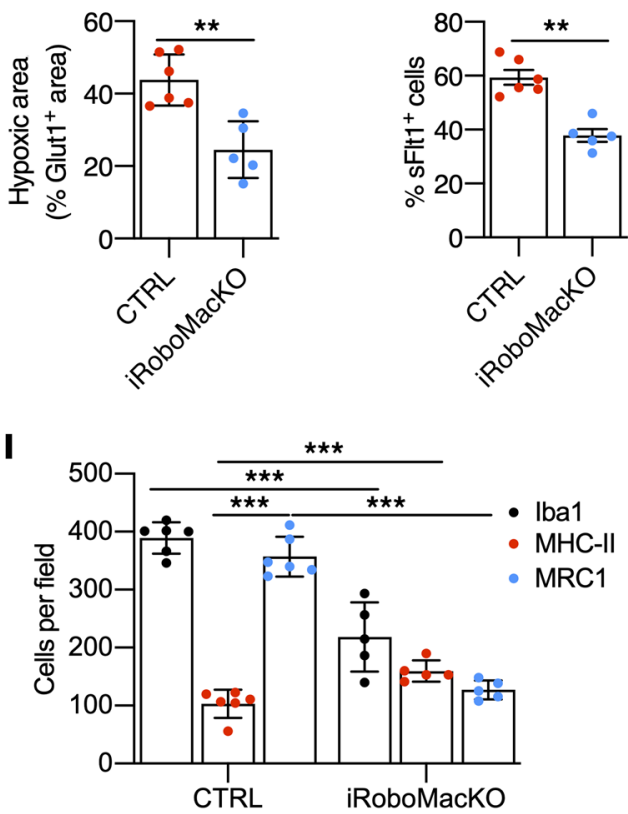

$\mathbf{N}$

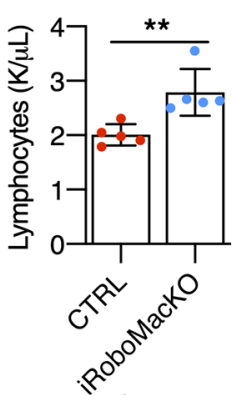

Day 14

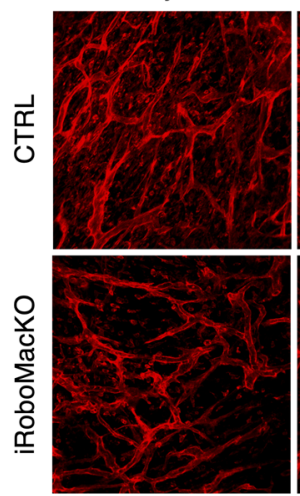

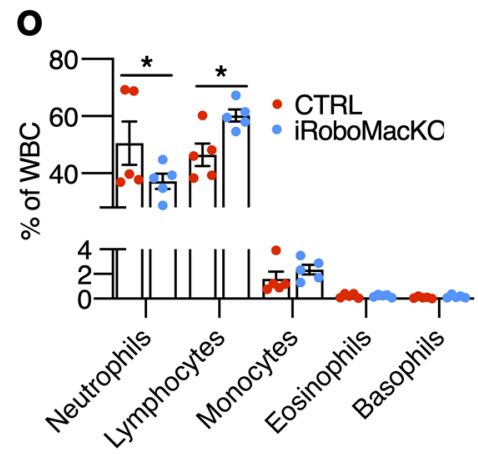

Day 21

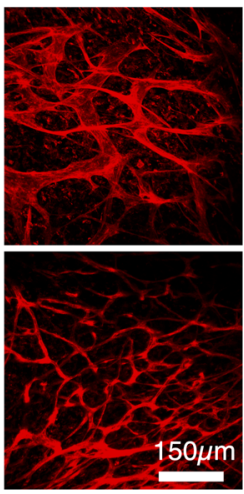

$\mathbf{K}$

$\mathbf{L}$

M
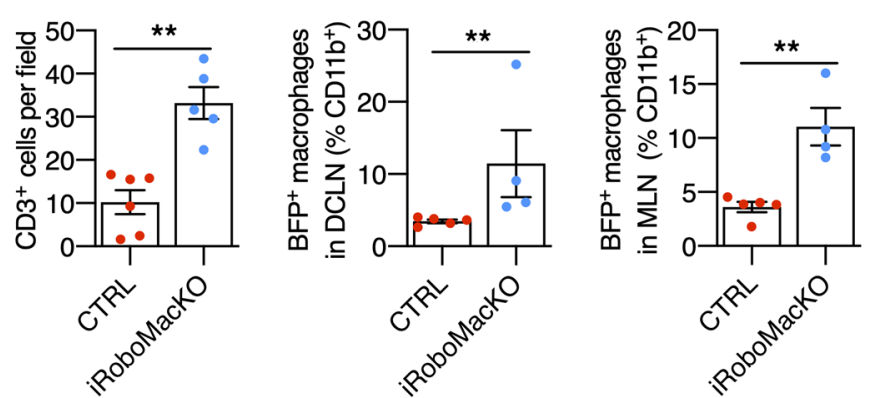

$\mathbf{P}$
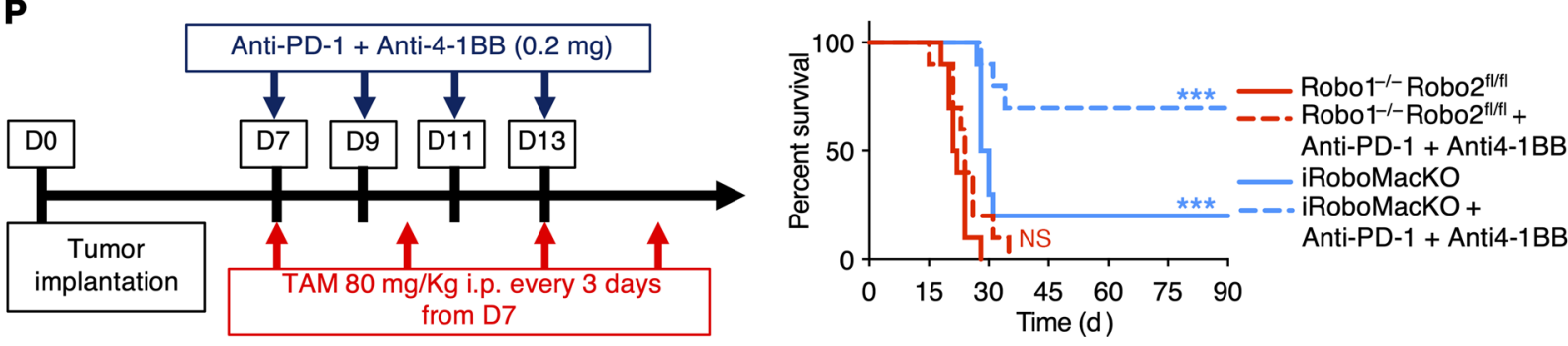
Figure 8. Macrophage-specific Robo1/2 knockout normalizes the TME. (A) MRI images of CTRL and iRoboMacKO mice 21 days after tumor implantation. (B and $\mathbf{C}$ ) Quantification of day 21 tumor size on MRI images (B, $n=4$ tumors per group, Mann-Whitney $U$ test) and serial vibratome sections (C, $n=7$ CTRL and 6 iRoboMacKO tumors, Mann-Whitney $U$ test). (D-F) In vivo 2-photon images of tumor-bearing mice (D) and quantification of vessel diameter $(\mathbf{E})$ and branchpoints $(\mathbf{F})(n=6$ mice per group, 1-way ANOVA). (G and $\mathbf{H}$ ) Glut1 (blue) immunohistochemistry on day 21 tumor-bearing mice (G), and quantification of tumor hypoxic areas $(\mathbf{H})(n=$ 6 CTRL and 5 iRoboMacKO tumors, Mann-Whitney $U$ test). (I-K) Quantification of F4/80, MHC-II, and MRC1+ cells (I), sFLT1 ${ }^{+} \mathrm{GFP}^{+}$cells (J), and total TALs $\left(\mathrm{CD3}^{+}\right)(\mathrm{K})(n=6 \mathrm{CTRL}$ and 5 iRoboMacKO tumors, 2-way ANOVA or Mann-Whitney $U$ test). (L and $\mathbf{M}$ ) FACS analysis of deep cervical DCLNs and MLNs from day 21 tumor-bearing mice ( $n=5$ CTRL and 4 iRoboMacKO mice; Mann-Whitney $U$ test). ( $\mathbf{N}$ and $\mathbf{O}$ ) Lymphocyte counts $(\mathbf{N})$ and differential WBC counts ( $\mathbf{0}$ ) from peripheral blood of day 21 tumor-bearing mice ( $n=5$ mice/group; Mann-Whitney $U$ test). (P) Eight-week-old mice engrafted with CT-2A BFP and treated with $80 \mathrm{mg} / \mathrm{kg}$ tamoxifen i.p. every 3 days starting 7 days after tumor implantation were randomly assigned to vehicle or anti-PD-1 + anti-4-1BB treatment ( $0.2 \mathrm{mg} /$ dose on days 7, 9, 11, and 13 after tumor implantation) ( $n=10 / 11$ mice per group, OS 21.5 days for Robo1 ${ }^{-/-}$Robo2 $2^{\mathrm{fl} / \mathrm{fl}}, 24$ days for Robo1 ${ }^{-/}$-Robo2 $2^{\mathrm{fl} / \mathrm{fl}}+$ anti-PD-1 + anti-4-1BB, 29 days for iRoboMacKO, and undetermined for iRoboMacKO + anti-PD-1 + anti-4-1BB; multiple comparisons log-rank test). Data are mean \pm SEM. ${ }^{*} P$ $<0.05,{ }^{* *} P<0.01,{ }^{* *} P<0.001$

The main findings of our study showed that SLIT2 expression levels correlated with tumor aggressiveness, poor prognosis, and immunosuppression. A limitation of the study is that the association between high SLIT2 expression and decreased survival in patients with GBM was not statistically significant within all the data sets analyzed.

Our data suggest that GBM tumor cells are a relevant source of SLIT2. Tumor cells constituted the majority of cells expressing SLIT2, and Slit2 knockdown in 2 murine GBM cell lines and in a human PDX model decreased tumor growth, while SLIT2 overexpression in CT2A cells enhanced murine GBM tumor growth. SLIT2 from other cell compartments could also affect GBM growth, but since genetic SLIT2 inhibition in tumor cells and systemic SLIT2 inhibition had similar effects in our mouse models, we conclude that tumor cell SLIT2 promotes GBM growth.

We observed that SLIT2 acted on different cell types within GBM. First, both human and mouse GBM tumor cells expressed ROBO1 and $\mathrm{ROBO} 2$ receptors. SLIT2 knockdown did not affect tumor cell proliferation or survival, and SLIT2 did not attract tumor cells in transwell assays in vitro. However, SLIT2 knockdown decreased tumor cell migration toward a serum gradient in transwell chambers and reduced spheroid invasion in fibrin gels, and patient-derived shSLIT2 GBM cells implanted in Nude mice decreased invasiveness compared with shCTRL. These results are consistent with SLIT2/ ROBO signaling driving proinvasive GBM tumor cell behavior in both mouse and patient-derived models. Our data contrast early studies with commercial human GBM cell lines where SLIT2/ROBO1 signaling inhibited migration $(51,52)$, but they support and extend studies using murine GBM models (55) and patient-derived tumor spheres and xenograft models (54), which showed that SLIT2/ROBO1 signaling in tumor cells promotes tumor invasiveness.

In addition to the tumor cells themselves, SLIT2 exerted major effects in the TME, and remarkably these changes appeared centered around $\mathrm{ROBO} 1$ and $\mathrm{ROBO} 2$ signaling in macrophages/microg- lial cells. We found that in the tumor context, $\mathrm{ROBO} 1$ and $\mathrm{ROBO} 2$ signaling inhibition in macrophages was sufficient to recapitulate all major aspects of tumor cell SLIT2 manipulation, or systemic SLIT2 inhibition with a ligand trap, and shifted the entire TME toward a normalized and cytotoxic phenotype. We identified 3 TME cell types that responded to tumor cell SLIT2, namely TAMs, endothelial cells of blood vessels and T cells. Genetic inhibition of Robo signaling in macrophages reduced macrophage recruitment to the TME, prevented phenotypic conversion into tumor-supportive macrophages, normalized tumor vasculature, and induced $\mathrm{T}$ cell-based antitumor responses. It remains possible that CSF1-R-CreERT2 leads to minor recombination in other immune cells, and that cell-autonomous Robo signaling in endothelial cells, which induces angiogenesis (28, 31), or T cell Robo signaling contribute to the observed effects in GBM, but macrophage Robo signaling appeared dominant.

Mechanistically, SLIT2-mediated TAM migration and polarization were $\mathrm{ROBO} 1$ and $\mathrm{ROBO} 2$ dependent and mediated by PI3K- $\gamma$ signaling. PI3K- $\gamma$ signaling inhibition has been previously shown to prevent TAM polarization and tumor progression in different cancer models (64), and this mechanism could be conserved in GBM. PI3K- $\gamma$ is traditionally activated by G proteincoupled receptors (GPCRs) or receptor tyrosine kinases (RTKs), therefore it remains to be established how ROBO activates PI3K- $\gamma$ mechanistically, via NCK-SOS activation of RAS or other small GTPases that can activate PI3K- $\gamma$ (67-69). Another possibility is that PI3K- $\gamma$ activation downstream of ROBO receptors depends on the coactivation of other RTKs or GPCRs and their endocytosis.

TAMs are the most abundant cells in the GBM microenvironment, and are known to contribute to immunosuppression in the $\operatorname{TME}(7-9,70)$ and dysmorphic angiogenesis $(16,71-73)$. Hence, TAMs are key players in the development of resistance to anticancer therapies $(17,74-77)$. Several attempts have been made to target TAM signaling for GBM treatment, including manipulation of VEGFa and angiopoietins Neuropilin1 $(78,79)$, CD47, or CSF-1R (80-82). Combined VEGF/angiopoietin inhibition led to vascular normalization and cytotoxic TAM polarization, but did not change $\mathrm{T}$ cell infiltration or activation profile $(83,84)$. CD47 inhibition prolonged GBM-bearing mice survival due to increased phagocytosis capacity and cytotoxic TAM polarization, but did not affect other components of the GBM microenvironment (85). CSF-1R inhibition did not change TAM production of proangiogenic molecules such as VEGFa and therefore did not lead to vascular normalization in GBM $(16,80,82)$. Hence, these strategies changed the TAM component of the GBM microenvironment, but they did not induce the profound changes in angiogenesis and $\mathrm{T}$ cell response achieved by SLIT2 inhibition. Systemic SLIT2 inhibition via intravenous injection of a SLIT2 ligand trap could be optimized and translated into clinical practice, especially for the treatment of patients with GBM with high SLIT2 expression.

\section{Methods}

A detailed discussion of the methods can be found in the Supplemental Material.

Animal procedures. All in vivo experiments were conducted in accordance to the European Community for experimental animal use guidelines (L358-86/609EEC). Animals were housed with free access to food and water in a 12-hour light/dark cycle. For survival experiments, mice 
A

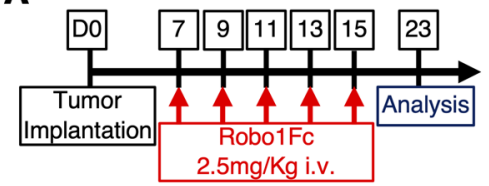

B

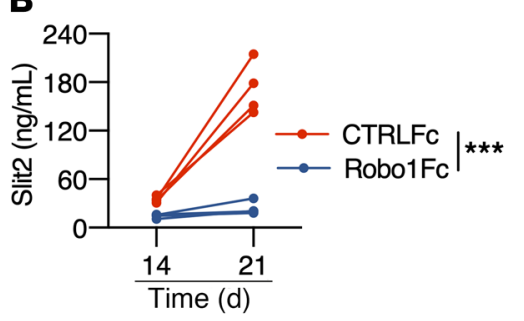

$\mathbf{F}$

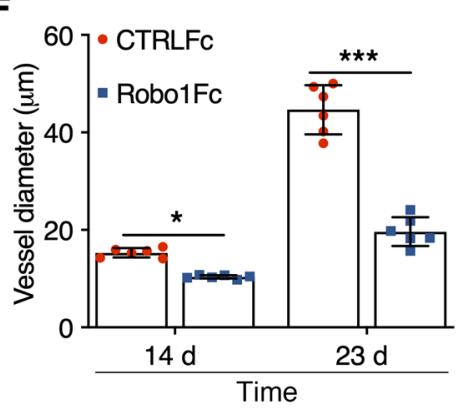

I

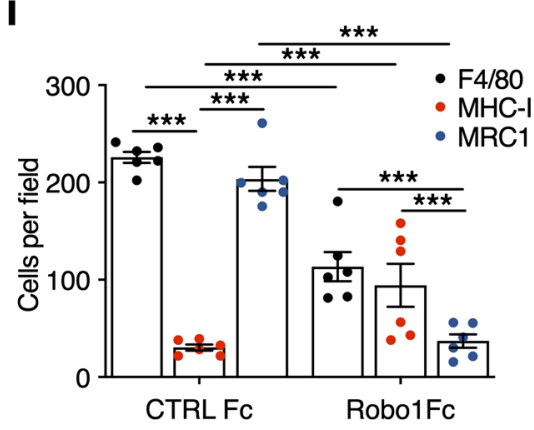

L
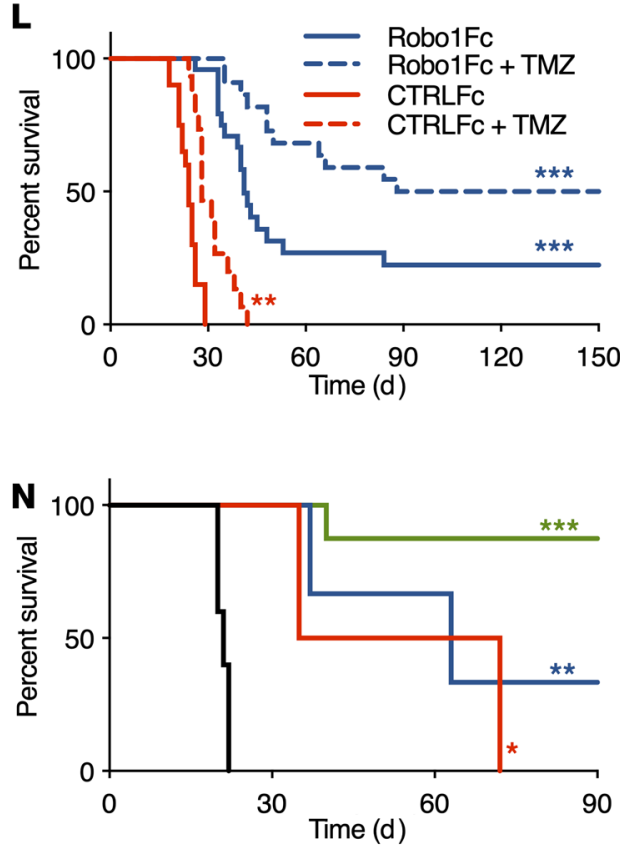

D
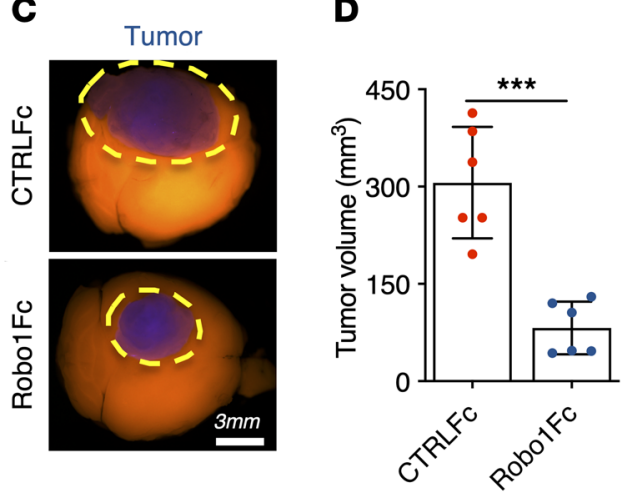

E

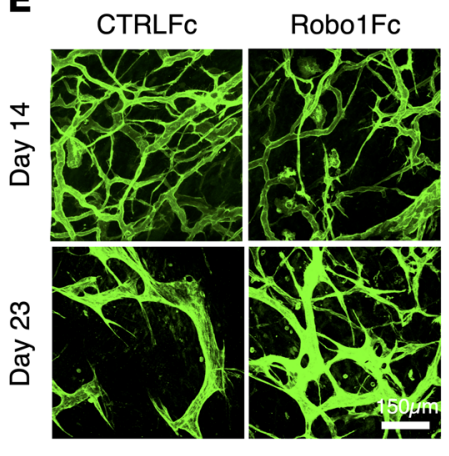

H

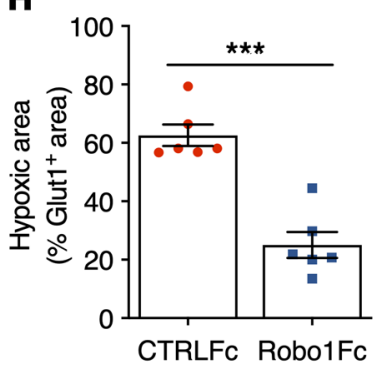

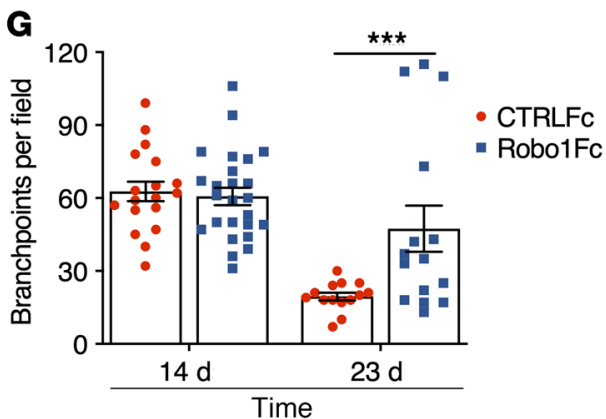

J

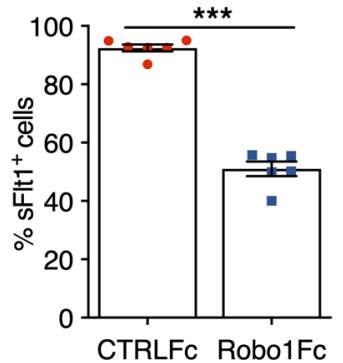

K
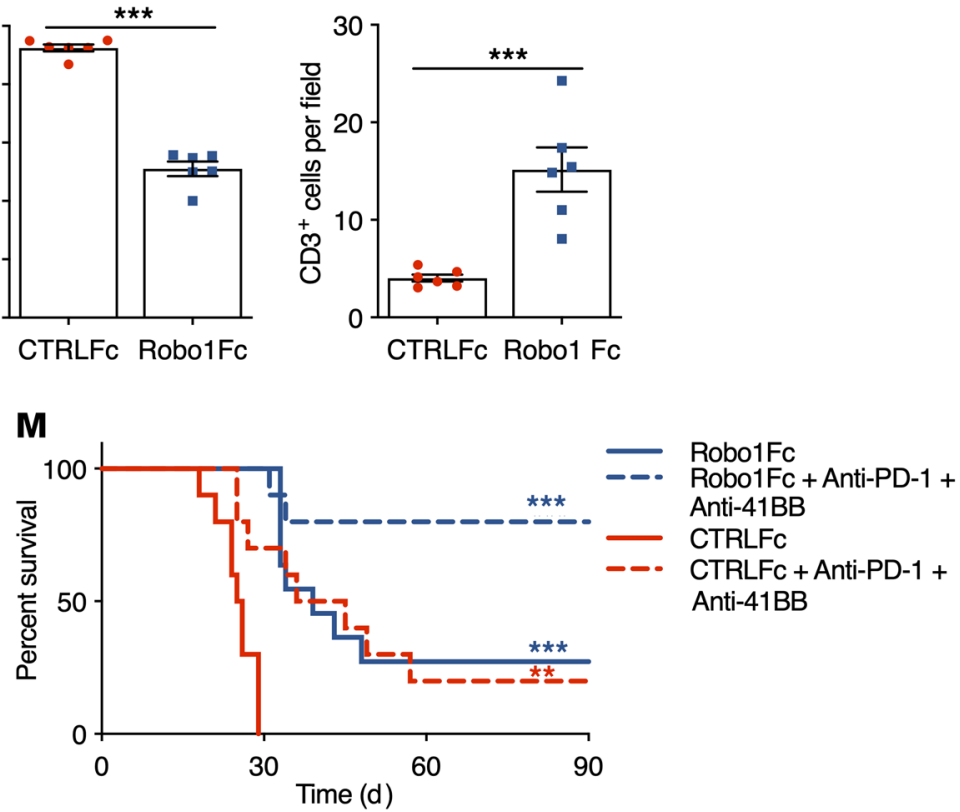
Figure 9. Robo1Fc treatment limits glioma growth. (A) Eight-week-old mice engrafted with CT-2A spheroids were treated with CTRLFc or Robo1Fc as indicated, and analyzed at day 23. (B) ELISA of serum Slit2 ( $n=4,2$-way ANOVA). (C and D) Tumor size at day 23 ( $n=6$, Student's $t$ test). (E-G) In vivo 2-photon images $(\mathbf{E})$ and quantification of vessel diameter $(\mathbf{F})$ and branchpoints (C) ( $n=6$ mice per group, 1-way ANOVA). (H) Quantification of Clut $1^{+}$area ( $n=6$ mice per group, Mann-Whitney $U$ test). (I-K) Quantification of F4/80, MHC-II, and MRC1 (I), soluble-Flt1 binding (J), and CD3 immunostaining (K) ( $n=6$ mice per group, 2-way ANOVA (I) or Student's $t$ test). (L) Eight-week-old tumor-bearing mice were assigned to CTRLFc + vehicle $(n=20)$, CTRLFc + TMZ $(n=15)$, Robo1Fc + vehicle $(n=24)$, or Robo1Fc + TMZ ( $n=22$; OS 24 days CTRLFc; 28 days CTRLFc + TMZ; 41 days Robo1Fc; 119 days Robo1Fc + TMZ; multiple comparisons Mantel-Cox log-rank). (M) Eight-week-old tumor-bearing mice were assigned to CTRLFC + vehicle, CTRLFc + anti-PD-1 + anti-4-1BB, Robo1Fc + vehicle, or Robo1Fc + anti-PD-1 + anti-4-1BB $(n=10 / 11$ mice per group; $0 S 25.5$ days CTRLFc; 40 days CTRLFc + anti-PD-1 + anti-4-1BB; 39 days Robo1Fc; and undetermined for Robo1Fc + anti-PD-1 + anti-4-1BB; multiple comparisons log-rank test). (N) Ninety days after tumor implantation, surviving mice from $\mathbf{M}(n=2$ anti-PD-1 + anti-4-1BB, $n=3$ Robo1Fc, and $n=8$ Robo1Fc + anti-PD-1 + anti-4-1BB) or 8-week-old tumor-naive mice $(n=10)$ were rechallenged in the contralateral hemisphere (OS 21 days naive mice; 53.5 days CTRLFc + anti-PD-1 + anti-4-1BB survivors; 63 days Robo1Fc survivors; and undetermined for Robo1Fc + anti-PD-1 + anti-4-1BB survivors; multiple comparisons log-rank test). Data are mean $\pm \mathrm{SEM} .{ }^{*} P<0.05,{ }^{* *} P$ $<0.01,{ }^{* * *} P<0.001$.

were euthanized if they exhibited signs of neurological morbidity or if they lost more than $20 \%$ of their body weight. C57bl6J and ROSA ${ }^{\mathrm{mT} / \mathrm{mG}}$ mice were used for survival and live imaging experiments, respectively. For generation of macrophage-specific Robo1/2 knockout, Robo1 ${ }^{-1}$ Robo2 $^{\mathrm{fl} / \mathrm{fl}}$ mice (28) were bred with CSF1-R-CreERT2, ROSA ${ }^{\mathrm{mT} / \mathrm{mG}}$ mice (65). Gene deletion was induced by injections of $80 \mathrm{mg} / \mathrm{kg}$ of tamoxifen every 2 days starting 7 days after tumor implantation and was verified on $\mathrm{GFP}^{+}$bone marrow monocytes/macrophages.

Live imaging. For multiphoton excitation of endogenous fluorophores in experimental gliomas, we used a Leica SP8 DIVE in vivo imaging system equipped with 4 Tune spectral external hybrid detectors and an InSightX3 laser (SpectraPhysics). The microscope was equipped with an in-house-designed mouse holding platform for intravital imaging (stereotactic frame, Narishige; gas anesthesia and body temperature monitoring/control, Minerve). Acquisition of ROSA ${ }^{\mathrm{mTmG}}$ reporter mice was performed at $1040 \mathrm{~nm}$ fixed wavelength. GFP signal from genetically modified tumor cells was acquired at $925 \mathrm{~nm}$ wavelength. Alexa Fluor 647-coupled Dextran was acquired at $1200 \mathrm{~nm}$ wavelength.

Statistics. For continuous variables, data are presented as mean \pm SEM. Results were considered significantly different if $P$ was less than 0.05. Between-group comparisons used the Mann-Whitney $U$ test or $t$ test, depending on the sample size for continuous variables. In cases where more than 2 groups were compared, 1-way ANOVA was performed, followed by Tukey's multiple comparison test. For comparisons involving grouped data, 2-way ANOVA was performed, followed by Tukey's multiple comparison test.

For survival experiments, log-rank (Mantel-Cox) tests and multiple comparison tests were performed. All the analyses were performed using Prism 6.0 software (GraphPad).

For mice in vivo imaging quantification, 4 to 9 fields per animal were pictured in the tumor center and blood vessel caliber, branching, and vessel perfusion were analyzed using Fiji software.
For mice ex vivo imaging quantification, 5 fields per individual were pictured in the tumor center and number of macrophages, overlapping stainings, hypoxic area, and tumor double-strand DNA damages were quantified using Fiji software.

Study approval. All in vivo experiments in animal models were conducted in accordance with the European Community for experimental animal use guidelines (L358-86/609EEC) with protocols (no. MESRI23570 and no. 175032018111214011311 v5) approved by the French Ministry of Higher Education, Research and Innovation. For the patient samples obtained at the Catholic University of Leuven, the study BRAIN-TUMOR-IMM-2014 (S57028) on human tissue was reviewed and approved by the Ethics Committee for Research UZ/KU Leuven. For the tumor samples obtained from the tumor bank "Onconeurotek" (certified NFS 96-900), written informed consent was obtained from the patients under approval of the institutional review boards (CPP-Ile de France IV-DC 2013-1962). These samples consisted of material in excess of what was required for diagnostic purposes. All subjects provided written informed consent prior to participation in the study.

\section{Author contributions}

LHG, AE, JLT, and TM conceived and designed the project. LHG, YX, LJ, LPF, RR, NM, MV, NL, CK, CL, TD, JR, TV, and TM performed experiments, collected and analyzed data. JD, LS, SDV and MS collected patient samples and provided clinical data for the study. BT, AB, AI, QRL, and FRSL contributed to study design and reviewed and revised the manuscript. FRSL, AE, and TM supervised the project. LHG, TM, and AE wrote the manuscript. All authors revised the manuscript.

\section{Acknowledgments}

This study was supported by the Fondation ARC pour la Recherche sur le Cancer-Institut National du Cancer (ARC-INCa) (to AE, JLT, and AB), Fondation pour la Recherche Medicale (DCP20171138945; to AE and BT), European Research Council (ERC) (grant agreement 834161 to AE), and the 'Coordenação de Aperfeiçoamento de Pessoal de Nível Superior' (CAPES), and the European Society of Cardiology (ESC Basic Research Fellowship) (to LHG). We thank the Paris Cardiovascular Research Center (PARCC) Flow and Image Cytometry facility; the France Life Imaging Network (grant ANR-11-INBS-0006) for its FDG-PET and MRI imaging platforms; and the 'Infrastructures Biologie Santé' (IBISA), Leducq Foundation (RETP: Visualisation des pathologies vasculaires) for its multiphoton imaging platform. We thank Holger Gerhardt for providing GL261, CT-2A, and RAW264.7 cells and CSF1-R-CreERT2 mice, and Alain Chedotal for providing the Robo1//-Robo $2^{\mathrm{f} / \mathrm{fl}}$ mice used in this study.

Address correspondence to: Anne Eichmann, Yale Cardiovascular Research Center (YCVRC), 300 George Street, 7th Floor, New Haven, Connecticut 06511, USA. Phone: 1.203.218.8459; Email: anne.eichmann@yale.edu. Or to: Thomas Mathivet, Paris Cardiovascular Research Center (PARCC), European Georges Pompidou Hospital, 56 Rue Leblanc, 75015 Paris, France. Phone: 33.1.53.98.80.18; Email: thomas.mathivet@inserm.fr. 
1. Ostrom QT, et al. CBTRUS statistical report: primary brain and other central nervous system tumors diagnosed in the United States in 20092013. Neuro Oncol.2016;18(Suppl_5):v1-v75.

2. Louis DN, et al. The 2016 World Health Organization classification of tumors of the central nervous system: a summary. Acta Neuropathol. 2016;131(6):803-820.

3. Omuro A, DeAngelis LM. Glioblastoma and other malignant gliomas: a clinical review. JAMA. 2013;310(17):1842-1850.

4. Aldape K, et al. Challenges to curing primary brain tumours. Nat Rev Clin Oncol. 2019;16(8):509-520.

5. Chen Z, et al. Cellular and molecular identity of tumor-associated macrophages in glioblastoma. Cancer Res. 2017;77(9):2266-2278.

6. Klemm F, et al. Interrogation of the microenvironmental landscape in brain tumors reveals disease-specific alterations of immune cells. Cell. 2020;181(7):1643-1660.

7. Quail DF, Joyce JA. The microenvironmental landscape of brain tumors. Cancer Cell. 2017;31(3):326-341.

8. Bloch $\mathrm{O}$, et al. Gliomas promote immunosuppression through induction of $\mathrm{B} 7-\mathrm{H} 1$ expression in tumor-associated macrophages. Clin Cancer Res. 2013;19(12):3165-3175.

9. Mitchem JB, et al. Targeting tumor-infiltrating macrophages decreases tumor-initiating cells, relieves immunosuppression, and improves chemotherapeutic responses. Cancer Res. 2013;73(3):1128-1141.

10. Caleb Rutledge W, et al. Tumor-infiltrating lymphocytes in glioblastoma are associated with specific genomic alterations and related to transcriptional class. Clin Cancer Res. 2013;19(18):4951-4960.

11. Chongsathidkiet $P$, et al. Sequestration of $T$ cells in bone marrow in the setting of glioblastoma and other intracranial tumors. Nat Med. 2018;24(9):1459-1468.

12. Lim M, et al. Current state of immunotherapy for glioblastoma. Nat Rev Clin Oncol. 2018;15(7):422-442.

13. Cloughesy TF, et al. Neoadjuvant anti-PD-1 immunotherapy promotes a survival benefit with intratumoral and systemic immune responses in recurrent glioblastoma. Nat Med. 2019;25(3):477-486.

14. Datta M, et al. Reprogramming the tumor microenvironment to improve immunotherapy: emerging strategies and combination therapies. Am Soc Clin Oncol Educ Book. 2019;39:165-174.

15. Carmeliet P, Jain RK. Principles and mechanisms of vessel normalization for cancer and other angiogenic diseases. Nat Rev Drug Discov. 2011;10(6):417-427.

16. Mathivet T, et al. Dynamic stroma reorganization drives blood vessel dysmorphia during glioma growth. EMBO Mol Med.2017;9(12):1629-1645.

17. Jain RK. Antiangiogenesis strategies revisited: from starving tumors to alleviating hypoxia. Cancer Cell. 2014;26(5):605-622.

18. Hambardzumyan D, et al. The role of microglia and macrophages in glioma maintenance and progression. Nat Neurosci. 2015;19(1):20-27.

19. Geraldo LHM, et al. Glioblastoma therapy in the age of molecular medicine. Trends Cancer. 2019;5(1):46-65.

20. Brose K, et al. Slit proteins bind Robo receptors and have an evolutionarily conserved role in repulsive axon guidance. Cell. 1999;96(6):795-806.

21. Kidd T, et al. Slit is the midline repellent for the robo receptor in Drosophila. Cell. 1999;96(6):785-794.

22. Blockus H, Chédotal A. Slit-Robo signaling. Development. 2016;143(17):3037-3044.

23. Barak R, et al. Structural principles in robo activation and auto-inhibition. Cell. 2019;177(2):272-285.

24. Koch AW, et al. Robo4 maintains vessel integrity and inhibits angiogenesis by interacting with UNC5B. Dev Cell. 2011;20(1):33-46.

25. Zelina P, et al. Signaling switch of the axon guidance receptor Robo3 during vertebrate evolution. Neuron. 2014;84(6):1258-1272.

26. Beamish IV, et al. Making connections: guidance cues and receptors at nonneural cell-cell junctions. Cold Spring Harb Perspect Biol. 2018;10(11):a029165

27. Bisiak F, McCarthy AA. Structure and function of roundabout receptors. Subcell Biochem. 2019;93:291-319.

28. Rama N, et al. Slit2 signaling through Robo1 and Robo 2 is required for retinal neovascularization. Nat Med. 2015;21(5):483-491.

29. Dubrac A, et al. Targeting NCK-mediated endothelial cell front-rear polarity inhibits neovascularization. Circulation. 2016;133(4):409-421.

30. Xu R, et al. Targeting skeletal endothelium to ameliorate bone loss. Nat Med. 2018;24(6):823-833.

31. Genet G, et al. Endophilin-A2 dependentVEGFR2 endocytosis promotes sprouting angiogenesis. Nat Commun. 2019;10(1):2650.

32. Pilling D, et al. Different isoforms of the neuronal guidance molecule Slit2 directly cause chemoattraction or chemorepulsion of human neutrophils. J Immunol. 2019;202(1):239-248.

33. Wu JY, et al. The neuronal repellent Slit inhibits leukocyte chemotaxis induced by chemotactic factors. Nature. 2001;410(6831):948-952.

34. Guan H, et al. Neuronal repellent Slit2 inhibits dendritic cell migration and the development of immune responses. Jimmunol. 2003;171(12):6519-6526.

35. Prasad A, et al. Slit-2/Robo-1 modulates the CXCL12/CXCR4-induced chemotaxis of T cells. JLeukoc Biol. 2007;82(3):465-476.

36 . Tole S, et al. The axonal repellent, Slit2, inhibits directional migration of circulating neutrophils. J Leukoc Biol. 2009;86(6):1403-1415.

37. Bhosle VK, et al. SLIT2/ROBO1-signaling inhibits macropinocytosis by opposing cortical cytoskeletal remodeling. Nat Commun. 2020;11(1):4112.

38. Wang B, et al. Induction of tumor angiogenesis by Slit-Robo signaling and inhibition of cancer growth by blocking Robo activity. Cancer Cell. 2003;4(1):19-29.

39. Wang LJ, et al. Targeting Slit-Roundabout signaling inhibits tumor angiogenesis in chemicalinduced squamous cell carcinogenesis. Cancer Sci. 2008;99(3):510-517.

40. Yang XM, et al. Slit-Robo signaling mediates lymphangiogenesis and promotes tumor lymphatic metastasis. Biochem Biophys Res Commun. 2010;396(2):571-577.

41. Schmid BC, et al. The neuronal guidance cue Slit2 induces targeted migration and may play a role in brain metastasis of breast cancer cells Breast Cancer Res Treat. 2007;106(3):333-342.

42. Zhou WJ, et al. Slit-Robo signaling induces malignant transformation through Hakai-mediated E-cadherin degradation during colorectal epithelial cell carcinogenesis. Cell Res. 2011;21(4):609-626.

43. Zhang QQ, et al. Slit2/Robo1 signaling promotes intestinal tumorigenesis through Src-mediated activation of the Wnt/ $\beta$-catenin pathway. Oncotarget. 2015;6(5):3123-3135.

44. Secq V, et al. Stromal SLIT2 impacts on pancreatic cancer-associated neural remodeling. Cell Death Dis. 2015;6(1):e1592

45. Zhao SJ, et al. SLIT2/ROBO1 axis contributes to the Warburg effect in osteosarcoma through activation of SRC/ERK/c-MYC/PFKFB2 pathway. Cell Death Dis. 2018;9(3):390.

46. Tavora B, et al. Tumoural activation of TLR3SLIT2 axis in endothelium drives metastasis. Nature. 2020;586(7828):299-304.

47. Zhou WJ, et al. Induction of intestinal stem cells by R-spondin 1 and Slit 2 augments chemoradioprotection. Nature. 2013;501(7465):107-111.

48. Dallol A, et al. SLIT2, a human homologue of the Drosophila Slit2 gene, has tumor suppressor activity and is frequently inactivated in lung and breast cancers. Cancer Res. 2002;62(20):5874-5880.

49. Tseng RC, et al. SLIT2 attenuation during lung cancer progression deregulates beta-catenin and E-cadherin and associates with poor prognosis. Cancer Res. 2010;70(2):543-551.

50. Chang PH, et al. Activation of Robo1 signaling of breast cancer cells by Slit 2 from stromal fibroblast restrains tumorigenesis via blocking PI3K/Akt/ $\beta$-catenin pathway. Cancer Res. 2012;72(18):4652-4661.

51. Mertsch S, et al. Slit2 involvement in glioma cell migration is mediated by Robo1 receptor. J Neurooncol. 2008;87(1):1-7.

52. Yiin JJ, et al. Slit2 inhibits glioma cell invasion in the brain by suppression of Cdc 42 activity. Neuro Oncol. 2009;11(6):779-789.

53. Liu L, et al. Slit2 and Robo1 expression as biomarkers for assessing prognosis in brain glioma patients. Surg Oncol. 2016;25(4):405-410.

54. Siebzehnrubl FA, et al. The ZEB1 pathway links glioblastoma initiation, invasion and chemoresistance. EMBO Mol Med. 2013;5(8):1196-1212.

55. Amodeo V, et al. A PML/Slit axis controls physiological cell migration and cancer invasion in the CNS. Cell Rep. 2017;20(2):411-426.

56. Verhaak RGW, et al. Integrated genomic analysis identifies clinically relevant subtypes of glioblastoma characterized by abnormalities in PDGFRA, IDH1, EGFR, and NF1. Cancer Cell. 2010;17(1):98-110.

57. Hartmann C, et al. Long-term survival in primary glioblastoma with versus without isocitrate dehydrogenase mutations. Clin Cancer Res. 2013;19(18):5146-5157.

58. Eckel-Passow JE, et al. Glioma groups based on 1p/19q, IDH, and TERT promoter mutations in tumors. N Engl J Med. 2015;372(26):2499-2508.

59. Christians A, et al. The prognostic role of IDH 
mutations in homogeneously treated patients with anaplastic astrocytomas and glioblastomas. Acta Neuropathol Commun. 2019;7(1):156.

60. Song E, et al. VEGF-C-driven lymphatic drainage enables immunosurveillance of brain tumours. Nature. 2020;577(7792):689-694.

61. Iwanami A, et al. PML mediates glioblastoma resistance to mammalian target of rapamycin (mTOR)-targeted therapies. Proc Natl Acad Sci U S A. 2013;110(11):4339-4344.

62. Garros-Regulez L, et al. Targeting SOX2 as a therapeutic strategy in glioblastoma. Front Oncol. 2016;6:222.

63. Woroniecka KI, et al. T-Cell dysfunction in glioblastoma: applying a new framework. Clin Cancer Res. 2018;24(16):3792-3802.

64. Kaneda MM, et al. PI3K $\gamma 3$ is a molecular switch that controls immune suppression. Nature. 2016;539(7629):437-442.

65. Qian BZ, et al. CCL2 recruits inflammatory monocytes to facilitate breast-tumour metastasis. Nature. 2011;475(7355):222-225.

66. Loubaki L, et al. In vivo depletion of leukocytes and platelets following injection of $\mathrm{T}$ cellspecific antibodies into mice. J Immunol Methods. 2013;393(1-2):38-44.

67. Yang L, Bashaw GJ. Son of sevenless directly links the Robo receptor to rac activation to control axon repulsion at the midline. Neuron. 2006;52(4):595-607.

68. Lucas B, Hardin J. Mind the (sr)GAP - roles of Slit-Robo GAPs in neurons, brains and beyond. JCell Sci. 2017;130(23):3965-3974.

69. Fritsch R, et al. XRAS and RHO families of GTPases directly regulate distinct phosphoinositide 3-kinase isoforms. Cell. 2013;153(5):1050-1063. 70. Pathria P, et al. Targeting tumor-associated macrophages in cancer. Trends Immunol. 2019;40(4):310-327.

71. Stockmann C, et al. Deletion of vascular endothelial growth factor in myeloid cells accelerates tumorigenesis. Nature. 2008;456(7223):814-818

72. Wenes M, et al. Macrophage metabolism controls tumor blood vessel morphogenesis and metastasis. Cell Metab. 2016;24(5):701-715.

73. Mazzone M, Bergers G. Regulation of blood and lymphatic vessels by immune cells in tumors and metastasis. Annu Rev Physiol. 2019;81:535-560.

74. Jain RK, et al. Angiogenesis in brain tumours. Nat Rev Neurosci. 2007;8(8):610-622.

75. De Palma M, Lewis CE. Macrophage regulation of tumor responses to anticancer therapies. Cancer Cell. 2013;23(3):277-286.

76. Jain RK. Normalizing tumor microenvironment to treat cancer: Bench to bedside to biomarkers. JClin Oncol. 2013;31(17):2205-2218.

77. Fukumura D, et al. Enhancing cancer immunotherapy using antiangiogenics: opportunities and challenges. Nat Rev Clin Oncol. 2018;15(5):325-340.

78. Miyauchi JT, et al. Ablation of neuropilin 1 from glioma-associated microglia and macrophages slows tumor progression. Oncotarget. 2016;7(9):9801-9814.

79. Miyauchi JT, et al. Deletion of neuropilin 1 from microglia or bone marrow-derived macrophages slows glioma progression. Cancer Res. 2018;78(3):685-694.

80. Pyonteck SM, et al. CSF-1R inhibition alters macrophage polarization and blocks glioma progression. Nat Med. 2013;19(10):1264-1272.

81. Ries CH, et al. Targeting tumor-associated macrophages with anti-CSF-1R antibody reveals a strategy for cancer therapy. Cancer Cell.
2014;25(6):846-859.

82. Quail DF, et al. The tumor microenvironment underlies acquired resistance to CSF-1R inhibition in gliomas. Science. 2016;352(6288):aad3018.

83. Kloepper J, et al. Ang-2/VEGF bispecific antibody reprograms macrophages and resident microglia to anti-tumor phenotype and prolongs glioblastoma survival. Proc Natl Acad Sci U S A. 2016;113(16):4476-4481.

84. Peterson TE, et al. Dual inhibition of Ang-2 and VEGF receptors normalizes tumor vasculature and prolongs survival in glioblastoma by altering macrophages. Proc Natl Acad Sci U S A. 2016;113(16):4470-4475.

85. Hutter G, et al. Microglia are effector cells of CD47-SIRP $\alpha$ antiphagocytic axis disruption against glioblastoma. Proc Natl Acad Sci U S A. 2019;116(3):997-1006.

86. Bowman RL, et al. GlioVis data portal for visualization and analysis of brain tumor expression datasets. Neuro Oncol. 2017;19(1):139-141.

87. Wang L, et al. The phenotypes of proliferating glioblastoma cells reside on a single axis of variation. Cancer Discov. 2019;9(12):1708-1719.

88. Neftel C, et al. An integrative model of cellular states, plasticity, and genetics for glioblastoma. Cell. 2019;178(4):835-849.

89. Darmanis S, et al. Single-cell RNA-seq analysis of infiltrating neoplastic cells at the migrating front of human glioblastoma. Cell Rep. 2017;21(5):1399-1410.

90. Stuart T, et al. Comprehensive integration of single-cell data. Cell. 2019;177(7):1888-1902.

91. Korsunsky I, et al. Fast, sensitive and accurate integration of single-cell data with Harmony. Nat Methods. 2019;16(12):1289-1296. 